\title{
A Semianalytical Three-Dimensional Elasticity Solution for Vibrations of Orthotropic Plates with Arbitrary Boundary Conditions
}

\author{
Jie Cui $(\mathbb{D}$, Zichao Li, Renchuan Ye $\mathbb{i}$, Wenan Jiang, and Shenghui Tao \\ School of Naval Architecture and Ocean Engineering, Jiangsu University of Science and Technology, Zhenjiang 212003, China \\ Correspondence should be addressed to Renchuan Ye; yrc@just.edu.cn
}

Received 14 April 2019; Accepted 9 June 2019; Published 25 July 2019

Academic Editor: Nerio Tullini

Copyright (C) 2019 Jie Cui et al. This is an open access article distributed under the Creative Commons Attribution License, which permits unrestricted use, distribution, and reproduction in any medium, provided the original work is properly cited.

\begin{abstract}
A semianalytical three-dimensional (3D) elasticity solution for the vibration of the orthotropic plate is presented under arbitrary boundary conditions. Three-dimensional (3D) elasticity theory provides the theoretical support for the energy function of orthotropic plates. The orthotropic plates which have the arbitrary boundary condition are realized by the way of arranging three sets of linear springs at the edges. With the aim of eliminating the nonsmooth phenomenon at the edges, the admissible displacement function of an orthotropic plate is expressed with a modified Fourier series solution. Under this framework, a change that occurs on the boundary conditions only needs to modify the boundary parameters of the orthotropic plate, without the need for new derivation, thus greatly saving the modeling time. The convergence and accuracy of the proposed method are better than those of the published literature. Lastly, the new vibration results and parametric research of thick orthotropic plates as well as the geometric parameter are also presented.
\end{abstract}

\section{Introduction}

As a basic building block, the orthotropic plates have been recognized and widely used by experts in various fields such as aerospace, architecture, shipbuilding, and other industries because of their good orthotropic mechanical properties such as strength to weight and high ratio of stiffness. In the above engineering applications, orthotropic rectangular plates as foundation components usually receive unknown loads. Thus, an intensive analysis about the vibration characteristics of the orthotropic rectangular plates has a beneficial effect on structural designers to carry out preliminary engineering design work. Compared with traditional isotropic materials, the vibration characteristics of orthotropic materials may be more complicated because of the material anisotropy. Traditional analytical and semianalytical numerical methods were used to solve the sandwich plate, laminated composite, and multilayered plates or functionally graded material plates with simply supported or clamped boundary conditions
[1-3]. Therefore, it is very urgent and necessary to develop a method to handle the orthotropic plates under the conditions of arbitrary boundary.

Up to now, characteristic of vibration about the orthotropic rectangular plate has attracted much attention and has achieved fruitful results. According to two variable refined plate theories, Thai and Kim [4-6] proposed a Levy-type solution for free vibration and bucking analysis of orthotropic plates having two opposite edges simply supported and the other two edges having arbitrary classical boundary conditions simultaneously. Sepahvand et al. [7] concluded a new plate theory about a study of the orthotropic plate under the condition of stochastic-free vibration with a method of generalized polynomial chaotic expansion. And an analytical homogenization model for torsion of orthotropic sandwich panels was proposed by Abbès and Guo [8]. Ghugal and Sayyad [9] proposed a concept of triangular shear deformation of the thick orthotropic plate to study the free vibration of this plate 
under classical boundary conditions. The vibration mechanism of orthotropic plates on nonlinear elastic foundations is given by Hsu [10] through the differential quadrature method. Bahmyari and Rahbar-Ranji [11] researched the thickness variation of a rectangular orthotropic plate on the nonuniform elastic foundation in one or two directions in the way of applying the meshless Galerkin method. In view of the classical plate theory (CPT), Valizadeh et al. [12] developed the isogeometric analysis to research buckling and free and forced vibration analyses to research orthotropic plates under the conditions of classical boundary. On the basis separation of the variables method, Xing and $\mathrm{Xu}$ [13] calculated the exact solution when the rectangular thin plates with orthotropic properties are free to vibrate and apply them to various classical boundary conditions. It was precise because of the rational use of the mixed first-order shear deformation plate theory that Zenkour [14] studied on the bending response about the simply supported orthotropic plate. Bakhadda and his coworkers [15-18] studied the free vibration, bending response, dynamic response of composite plates, and FGM plates by various valuable shear deformation theories. Papkov and Banerjee [19] proposed a new solution, which aims to solve the free vibration and buckling analysis of orthotropic rectangular plates under the different classical boundary conditions. And the characteristics of the orthotropic rectangular plate under free vibration are studied by Liu et al. [20]; in particular, the plates undergo free vibration in one or two directions. Through the Rayleigh-Ritz procedure, Shi et al. [21] studied a special plane vibration analysis and the special point is that the elastic boundary constraints of the orthotropic rectangular plates are nonuniform as well as internally supported. An effective hybrid method proposed by Jafari and Eftekhari [22] is suitable for the free vibration and buckling analysis about the orthotropic rectangular plate under various classical boundary conditions. Liu et al. $[23,24]$ proposed an accurate closedform solution regarding orthotropic rectangular Mindlin plates to solve the free vibration problem under some classical boundary conditions. Chang and Tarn [25] researched the $3 \mathrm{D}$ analysis associated with orthotropic rectangular plates which supported external loads from the top and bottom surfaces by using the state space approach. In the aspect of 3D analysis, Teo and Liew [26] found that a method called differential quadrature (DQ) can effectively analyze rectangular orthotropic plates which have the boundary conditions of SSSS, SCCC, CSSS, and CCCC. Tian et al. [27, 28] proposed a new higher order analysis model for sandwich and FGM plates with flexible core and obtained the fundamental frequencies and static bending under sinusoidal and uniformly distributed loads. A semianalytical method is employed to analyze free vibration behaviors of composite laminated doubly curved, cylindrical, and spherical shells by Li et al. [29-31]. Abdelaziz and his coworkers [32-34] analyzed free vibration, bending, buckling, and postbuckling of FGM sandwich plates and nanobeams with various boundary conditions by various shear deformation theories. Cui et al. [35] investigated energy absorption of double layered and Sandwich plates under ballistic impact via experiment and numerical simulation. Liu et al. [36] assessed the material strain rate effects on steel plates under lateral impact loads.

The above results have been clearly described. It does not have trouble in finding that most of the current research results are confined to thin orthotropic plates and moderately thick orthotropic plates. In their research, they are based on the two-dimensional (2D) elasticity theory. From existing literature and materials, we can see that twodimensional elasticity theories are based on assumptions. However, due to the existence of the assumption, the computation results are not credible at high modes. Based on this background, the three-dimensional theory is developed. And its results are generally accepted by researchers and used as contrastive data for reference in twodimensional elasticity theory. However, for the greatest knowledge of the author, only two papers are currently committed to research the $3 \mathrm{D}$ vibration analysis of thick orthotropic plates that the conditions are constrained within the classical boundary.

A $3 \mathrm{D}$ vibration analysis of thick orthotropic plates under the general boundary condition is firstly performed in this paper. The theoretical formula is obtained by using this three-dimensional elastic theory. The arbitrary boundary conditions of the orthotropic plates are achieved through arranging three sets of linear springs at the edges. No matter how the boundary conditions are, the admissible displacement function of a thick orthotropic plate is uniformly expressed with improved Fourier series. Under this framework, when the boundary conditions of the orthotropic plates change, only the boundary parameters need to be modified, without the need for new derivation, thus greatly saving the modeling time. On this foundation, the astringency and exactitude of existing methods were verified by contrasting the results of existing literature and data. Not limited to this, some new numerical results are further displayed as reference data for future data validation.

\section{Theoretical Formulations}

2.1. Description of the Thick Orthotropic Plates. Figure 1 shows a thick orthotropic plate under the conditions of general boundary. The coordinate system $(x, y, z)$ of the thick orthotropic plate is located at the bottom. $a, b$, and $h$ represent length, width, and height successively. The symbols $u, v$, and $w$, respectively, represent displacement components in the length, width, and thickness directions. As shows in Figure 1(b), three kinds of linear springs, i.e., linear elastic $k_{u}$ in the direction of length, linear elastic $k_{v}$ in the direction of width, and linear elastic $k_{w}$ in the direction of thickness, are uniformly arranged at the structural boundaries. By simply setting the stiffness coefficient, the real boundary force can be simulated and 


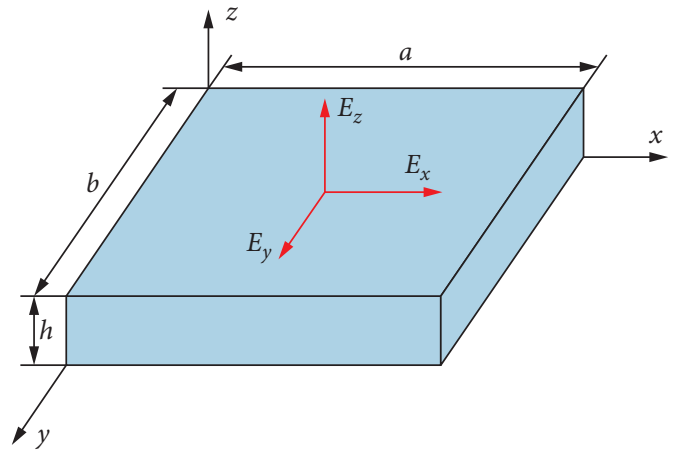

(a)

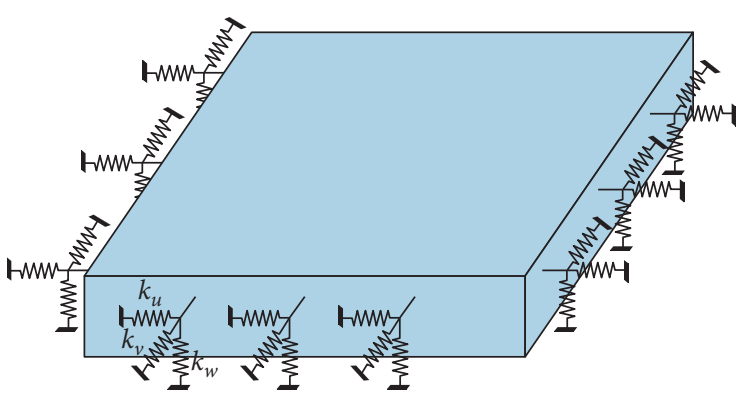

(b)

FIGURE 1: Schematic diagram of the 3D orthotropic rectangular plates: (a) geometry and coordinates; (b) boundary restraining springs.

the equivalent ideal boundary condition is available. Each boundary spring stiffness values are set to infinity $\left(k_{u}=k_{v}=k_{w}=10^{16}\right)$; for instance, it becomes very easy to obtain clamped boundary conditions.

2.2. Energy Expression of Thick Orthotropic Plates. On account of the 3D elastic theory, the linear strain-displacement regulations of thick orthotropic plates are given as follows:

$$
\begin{aligned}
\varepsilon_{x x} & =\frac{\partial u}{\partial x}, \\
\varepsilon_{y y} & =\frac{\partial v}{\partial y}, \\
\varepsilon_{z z} & =\frac{\partial w}{\partial z}, \\
\gamma_{x y} & =\frac{\partial u}{\partial y}+\frac{\partial v}{\partial x}, \\
\gamma_{x z} & =\frac{\partial u}{\partial z}+\frac{\partial w}{\partial x}, \\
\gamma_{y z} & =\frac{\partial v}{\partial z}+\frac{\partial w}{\partial y} .
\end{aligned}
$$

The stress of a thick orthotropic plates constrained by linear elastic theory can be defined as follows:

$$
\left\{\begin{array}{c}
\sigma_{x x} \\
\sigma_{y y} \\
\sigma_{z z} \\
\sigma_{x y} \\
\sigma_{x z} \\
\sigma_{y z}
\end{array}\right\}=\left[\begin{array}{cccccc}
C_{11} & C_{12} & C_{13} & 0 & 0 & 0 \\
C_{12} & C_{22} & C_{23} & 0 & 0 & 0 \\
C_{13} & C_{23} & C_{33} & 0 & 0 & 0 \\
0 & 0 & 0 & C_{66} & 0 & 0 \\
0 & 0 & 0 & 0 & C_{55} & 0 \\
0 & 0 & 0 & 0 & 0 & C_{44}
\end{array}\right]\left\{\begin{array}{c}
\varepsilon_{x x} \\
\varepsilon_{y y} \\
\varepsilon_{z z} \\
\gamma_{x y} \\
\gamma_{x z} \\
\gamma_{y z}
\end{array}\right\},
$$

where stiffness coefficients $C_{i j}$ are obtained as below:

$$
\begin{aligned}
& C_{11}=\eta E_{x}\left(1-\mu_{y z} \mu_{z y}\right), \\
& C_{12}=\eta E_{y}\left(\mu_{x y}+\mu_{x z} \mu_{z y}\right), \\
& C_{22}=\eta E_{y}\left(1-\mu_{x z} \mu_{z x}\right), \\
& C_{23}=\eta E_{z}\left(\mu_{y z}+\mu_{y x} \mu_{x z}\right), \\
& C_{33}=\eta E_{z}\left(1-\mu_{x y} \mu_{y x}\right), \\
& C_{31}=\eta E_{x}\left(\mu_{z x}+\mu_{z y} \mu_{y x}\right), \\
& C_{44}=G_{y z}, \\
& C_{55}=G_{x z}, \\
& C_{66}=G_{x y}, \\
& \eta=\frac{1}{1-\mu_{x y} \mu_{y x}-\mu_{y z} \mu_{z y}-\mu_{z x} \mu_{x z}-\mu_{x y} \mu_{y z} \mu_{z x}-\mu_{y x} \mu_{x z} \mu_{z y}}, \\
& \mu_{x y} E_{y}=\mu_{y x} E_{x}, \\
& \mu_{x z} E_{z}=\mu_{z x} E_{x}, \\
& \mu_{z y} E_{y}=\mu_{y z} E_{z} .
\end{aligned}
$$

It must be emphasized here that when the above material parameters are simplified to isotropic materials, a thick isotropic plate can be obtained directly, and their stiffness coefficients can be simplified as follows: 


$$
\begin{aligned}
C_{11} & =C_{22}=C_{33}=\lambda+2 G, \\
C_{12} & =C_{13}=C_{21}=C_{23}=C_{31}=C_{32}=\lambda, \\
C_{44} & =C_{55}=C_{66}=G, \\
\lambda & =\frac{E \mu}{(1+\mu)(1-2 \mu)}, \\
G & =\frac{E}{2(1+\mu)} .
\end{aligned}
$$

Next, the $U$ representing the strain energy of the thick orthotropic plates which is based on the elastic theory is as follows:

$$
\begin{aligned}
U= & \frac{1}{2} \int_{0}^{h} \int_{0}^{a} \int_{0}^{b}\left(\sigma_{x x} \varepsilon_{x x}+\sigma_{y y} \varepsilon_{y y}+\sigma_{z z} \varepsilon_{z z}+\sigma_{x y} \varepsilon_{x y}+\sigma_{x z} \varepsilon_{x z}\right. \\
& \left.+\sigma_{y z} \varepsilon_{y z}\right) d x d y d z \\
= & \frac{1}{2} \int_{0}^{h} \int_{0}^{a} \int_{0}^{b}\left(C_{11}\left(\frac{\partial u}{\partial x}\right)^{2}+C_{22}\left(\frac{\partial v}{\partial y}\right)^{2}+C_{33}\left(\frac{\partial w}{\partial z}\right)^{2}\right. \\
& +2\left(C_{12} \frac{\partial u}{\partial x} \frac{\partial v}{\partial y}+C_{13} \frac{\partial u}{\partial x} \frac{\partial w}{\partial z}+C_{23} \frac{\partial v}{\partial y} \frac{\partial w}{\partial z}\right) \\
& +\left[C_{66}\left(\frac{\partial u}{\partial y}\right)^{2}+C_{66}\left(\frac{\partial v}{\partial x}\right)^{2}+C_{55}\left(\frac{\partial u}{\partial z}\right)^{2}+C_{55}\left(\frac{\partial w}{\partial x}\right)^{2}\right. \\
& \left.+C_{44}\left(\frac{\partial v}{\partial z}\right)^{2}+C_{44}\left(\frac{\partial w}{\partial y}\right)^{2}\right] \\
& \left.+2\left(C_{66} \frac{\partial u}{\partial y} \frac{\partial v}{\partial x}+C_{55} \frac{\partial u}{\partial z} \frac{\partial w}{\partial x}+\frac{\partial v}{\partial z} \frac{\partial w}{\partial y}\right)\right)^{2} d x d y d z
\end{aligned}
$$

The $T$ representing kinetic energy [37] about thick orthotropic plates is as follows:

$$
T=\frac{1}{2} \int_{0}^{h} \int_{0}^{a} \int_{0}^{b} \rho\left[\left(\frac{\partial u}{\partial t}\right)^{2}+\left(\frac{\partial v}{\partial t}\right)^{2}+\left(\frac{\partial w}{\partial t}\right)^{2}\right] d x d y d z
$$

Boundary conditions are efficiently simulated by artificial spring boundary technology. The actual operation is based on the introduction of three types of springs at the boundary to correspond to the actual boundary forces. Therefore, $U_{\text {sp }}$ representing the potential energy which is located in the boundary spring can be expressed as follows:

$$
\begin{aligned}
U_{\mathrm{sp}}= & \frac{1}{2} \int_{0}^{h} \int_{0}^{b}\left\{\left.\left[k_{x 0}^{u} u^{2}+k_{x 0}^{v} v^{2}+k_{x 0}^{w} w^{2}\right]\right|_{x=0}\right. \\
& \left.+\left.\left[k_{x 1}^{u} u^{2}+k_{x 1}^{v} v^{2}+k_{x 1}^{w} w^{2}\right]\right|_{x=a}\right\} d y d z \\
& +\frac{1}{2} \int_{0}^{h} \int_{0}^{a}\left\{\left.\left[k_{y 0}^{u} u^{2}+k_{y 0}^{v} v^{2}+k_{y 0}^{w} w^{2}\right]\right|_{y=0}\right. \\
& \left.+\left.\left[k_{y 1}^{u} u^{2}+k_{y 1}^{v} v^{2}+k_{y 1}^{w} w^{2}\right]\right|_{y=b}\right\} d x d z .
\end{aligned}
$$

The Lagrangian function of the thick orthotropic plates obtained from the above expression is expressed as follows:

$$
L=T-U-U_{\mathrm{sp}}
$$

2.3. Admissible Displacement Functions. The main task of this chapter is to establish admissible displacement function of the thick plate displacement when using the Ritz method. According to the previous research, it is easy to find that the traditional displacement function has some shortcomings. Therefore, in this paper, we will make use of the modified Fourier series proposed by the author before. The principle and mathematical analysis process of the modified Fourier series solution are included in [38-41]. Therefore, on the basis of the improved series solution principle and the motion equation, the displacement tolerance function of the thick orthotropic plate is uniformly described as a periodic function:

$$
\begin{aligned}
& u(x, y, z)=\left(\mathbf{U}^{\Omega}(x, y, z)+\sum_{q=1}^{6} \mathbf{U}_{q}^{\mathrm{S}}(x, y, z)\right) \mathbf{A}_{m n q}, \\
& v(x, y, z)=\left(\mathbf{V}^{\Omega}(x, y, z)+\sum_{q=1}^{6} \mathbf{V}_{q}^{\mathrm{S}}(x, y, z)\right) \mathbf{B}_{m n q}, \\
& w(x, y, z)=\left(\mathbf{W}^{\Omega}(x, y, z)+\sum_{q=1}^{6} \mathbf{W}_{q}^{\mathrm{S}}(x, y, z)\right) \mathbf{C}_{m n q},
\end{aligned}
$$

where $\mathbf{U}^{\Omega}, \mathbf{V}^{\Omega}$, and $\mathbf{W}^{\Omega}$ are representative of the internal displacement distribution function about the thick orthotropic plate and $\mathbf{U}_{q}^{S}, \mathbf{V}_{q}^{S}$, and $\mathbf{W}_{q}^{S}$ represent the complementary sequences of the displacement on the boundaries of the thick orthotropic plates. And the three 3D Fourier coefficients vector are $\mathbf{A}_{m n q}, \mathbf{B}_{m n q}$, and $\mathbf{C}_{m n q}$, respectively. The following formulas represent the specific elements of each vector symbol:

$$
\begin{aligned}
\mathbf{U}^{\Omega}= & \mathbf{V}^{\Omega}=\mathbf{W}^{\Omega}=\left\{\cos \lambda_{0}^{a} x \cos \lambda_{0}^{b} y \cos \lambda_{0}^{h} z, \cdots, \cos \lambda_{0}^{a} x \cos \lambda_{0}^{b} y \cos \lambda_{Q_{1}}^{h} z, \ldots, \cos \lambda_{0}^{a} x \cos \lambda_{N_{1}}^{b} y\right. \\
& \left.\cdot \cos \lambda_{Q_{1}}^{h} z, \ldots, \cos \lambda_{M_{1}}^{a} x \cos \lambda_{N_{1}}^{b} y \cos \lambda_{Q_{1}}^{h} z\right\},
\end{aligned}
$$




$$
\begin{aligned}
& \mathbf{U}_{1}^{\mathrm{B}}=\mathbf{V}_{1}^{\mathrm{B}}=\mathbf{W}_{1}^{\mathrm{B}}=\left\{\sin \left(\lambda_{-2}^{a} x\right) \cos \left(\lambda_{0}^{b} y\right), \ldots, \sin \left(\lambda_{-2}^{a} x\right) \cos \left(\lambda_{n}^{b} y\right), \ldots, \sin \left(\lambda_{-2}^{a} x\right)\right. \\
& \left.\cdot \cos \left(\lambda_{N}^{b} y\right), \ldots, \sin \left(\lambda_{-1}^{a} x\right) \cos \left(\lambda_{N}^{b} y\right)\right\} \text {, } \\
& \mathbf{U}_{2}^{\mathrm{B}}=\mathbf{V}_{2}^{\mathrm{B}}=\mathbf{W}_{2}^{\mathrm{B}}=\boldsymbol{\Phi}_{2}^{\mathrm{B}}=\boldsymbol{\Theta}_{2}^{\mathrm{B}}=\left\{\cos \left(\lambda_{0}^{a} x\right) \sin \left(\lambda_{-2}^{b} y\right), \cos \left(\lambda_{0}^{a} x\right) \sin \left(\lambda_{-1}^{b} y\right), \ldots, \cos \left(\lambda_{m}^{a} x\right)\right. \\
& \left.\cdot \sin \left(\lambda_{-2}^{b} y\right), \ldots, \cos \left(\lambda_{M}^{a} x\right) \sin \left(\lambda_{-1}^{b} y\right)\right\} \\
& \mathbf{U}_{1}^{\mathrm{S}}=\mathbf{V}_{1}^{\mathrm{S}}=\mathbf{W}_{1}^{\mathrm{S}}=\left\{\sin \lambda_{-2}^{a} x \cos \lambda_{0}^{b} y \cos \lambda_{0}^{h} z, \ldots, \sin \lambda_{-2}^{a} x \cos \lambda_{0}^{b} y \cos \lambda_{\mathrm{Q}_{1}}^{h} z, \ldots, \sin \lambda_{-2}^{a} x\right. \\
& \left.\cdot \cos \lambda_{N_{1}}^{b} y \cos \lambda_{\mathrm{Q}_{1}}^{h} z, \ldots, \sin \lambda_{-1}^{a} x \cos \lambda_{N_{1}}^{b} y \cos \lambda_{\mathrm{Q}_{1}}^{h} z\right\} \text {, } \\
& \mathbf{U}_{2}^{\mathrm{S}}=\mathbf{V}_{2}^{\mathrm{S}}=\mathbf{W}_{2}^{\mathrm{S}}=\left\{\cos \lambda_{0}^{a} x \sin \lambda_{-2}^{b} y \cos \lambda_{0}^{h} z, \ldots, \cos \lambda_{0}^{a} x \sin \lambda_{-2}^{b} y \cos \lambda_{\mathrm{Q}_{1}}^{h} z, \ldots, \cos \lambda_{0}^{a} x\right. \\
& \left.\cdot \sin \lambda_{-2}^{b} y \cos \lambda_{\mathrm{Q}_{1}}^{h} z, \ldots, \cos \lambda_{M_{1}}^{a} x \sin \lambda_{-1}^{b} y \cos \lambda_{\mathrm{Q}_{1}}^{h} z\right\}, \\
& \mathbf{U}_{3}^{\mathrm{S}}=\mathbf{V}_{3}^{\mathrm{S}}=\mathbf{W}_{3}^{\mathrm{S}}=\left\{\cos \lambda_{0}^{a} x \cos \lambda_{0}^{b} y \sin \lambda_{-2}^{h} z, \cos \lambda_{0}^{a} x \cos \lambda_{0}^{b} y \sin \lambda_{-1}^{h} z, \ldots, \cos \lambda_{0}^{a} x \cos \lambda_{N_{1}}^{b} y\right. \\
& \left.\cdot \sin \lambda_{-2}^{h} z, \ldots, \cos \lambda_{M_{1}}^{a} x \cos \lambda_{N_{1}}^{b} y \sin \lambda_{-1}^{h} z\right\} \text {, } \\
& \mathbf{U}_{4}^{\mathrm{S}}=\mathbf{V}_{4}^{\mathrm{S}}=\mathbf{W}_{4}^{\mathrm{S}}=\left\{\sin \lambda_{-2}^{a} x \sin \lambda_{-2}^{b} y \cos \lambda_{0}^{h} z, \ldots, \sin \lambda_{-2}^{a} x \sin \lambda_{-2}^{b} y \cos \lambda_{\mathrm{Q}_{1}}^{h} z, \ldots, \sin \lambda_{-2}^{a} x\right. \\
& \left.\cdot \sin \lambda_{-1}^{b} y \cos \lambda_{\mathrm{Q}_{1}}^{h} z, \ldots, \sin \lambda_{-1}^{a} x \sin \lambda_{-1}^{b} y \cos \lambda_{\mathrm{Q}_{1}}^{h} z\right\}, \\
& \mathbf{U}_{5}^{\mathrm{S}}=\mathbf{V}_{5}^{\mathrm{S}}=\mathbf{W}_{5}^{\mathrm{S}}=\left\{\sin \lambda_{-2}^{a} x \cos \lambda_{0}^{b} y \sin \lambda_{-2}^{h} z, \sin \lambda_{-2}^{a} x \cos \lambda_{0}^{b} y \sin \lambda_{-1}^{h} z, \ldots, \sin \lambda_{-2}^{a} x\right. \\
& \left.\cdot \cos \lambda_{N_{1}}^{b} y \sin \lambda_{-2}^{h} z, \ldots, \sin \lambda_{-1}^{a} x \cos \lambda_{N_{1}}^{b} y \sin \lambda_{-1}^{h} z\right\}, \\
& \mathbf{U}_{6}^{S}=\mathbf{V}_{6}^{S}=\mathbf{W}_{6}^{S}=\left\{\cos \lambda_{0}^{a} x \sin \lambda_{-2}^{b} y \sin \lambda_{-2}^{h} z, \cos \lambda_{0}^{a} x \sin \lambda_{-2}^{b} y \sin \lambda_{-1}^{h} z, \cos \lambda_{0}^{a} x \sin \lambda_{-1}^{b} y\right. \\
& \left.\cdot \sin \lambda_{-2}^{h} z, \ldots, \cos \lambda_{M_{1}}^{a} x \sin \lambda_{-1}^{b} y \sin \lambda_{-1}^{h} z\right\}, \\
& \Delta_{m_{1} n_{1} q_{1}}=\left\{\Delta_{0,0,0}^{1}, \ldots, \Delta_{0,0, q_{1}}^{1}, \ldots, \Delta_{0,0, Q_{1}}^{1}, \ldots, \Delta_{0, N_{1}, Q_{1}}^{1}, \ldots, \Delta_{m_{1}, n_{1}, q^{2}}^{1}, \ldots, \Delta_{M_{1}, N_{1}, Q_{1}}^{1},\right. \\
& \Delta_{-2,0,0}^{2}, \ldots, \Delta_{-2,0, q_{1}}^{2}, \ldots, \Delta_{-2,0, Q_{1}}^{2}, \ldots, \Delta_{-2, n_{1}, q_{1}}^{2}, \ldots, \Delta_{-1, n_{1}, q_{1}}^{2}, \ldots, \Delta_{-1, N_{1}, Q_{1}}^{2} \\
& \Delta_{0,-2,0}^{3}, \ldots, \Delta_{0,-2, q_{1}}^{3}, \ldots, \Delta_{0,-2, Q_{1}}^{3}, \cdots, \Delta_{m_{1},-2, q_{1}}^{3}, \cdots, \Delta_{m_{1},-1, q_{1}}^{3}, \ldots, \Delta_{M_{1},-1, Q_{1}}^{3} \\
& \Delta_{0,0,-2}^{4}, \Delta_{0,0,-1}^{4}, \ldots, \Delta_{0, N_{1},-2}^{4}, \ldots, \Delta_{m_{1}, n_{1},-2}^{4}, \ldots, \Delta_{m_{1}, n_{1},-1}^{4}, \ldots, \Delta_{M_{1}, N_{1},-1}^{4} \text {, } \\
& \Delta_{-2,-2,0}^{5}, \ldots, \Delta_{-2,-2, q_{1}}^{5}, \cdots, \Delta_{-2,-2, Q_{1}}^{5}, \ldots, \Delta_{-2,-1, q_{1}}^{5}, \ldots, \Delta_{-2,-1, Q_{1}}^{5}, \ldots, \Delta_{-1,-1, Q_{1}}^{5}, \\
& \Delta_{-2,0,-2}^{6}, \Delta_{-2,0,-1}^{6}, \ldots, \Delta_{-2, n_{1},-2}^{6}, \ldots, \Delta_{-2, N_{1},-2}^{6}, \ldots, \Delta_{-1, n_{1}, Q_{1}}^{6}, \ldots, \Delta_{-1, N_{1},-1}^{6} \text {, } \\
& \left.\Delta_{0,-2,-2}^{7}, \Delta_{0,-2,-1}^{7}, \ldots, \Delta_{m_{1},-2,-2}^{7}, \ldots, \Delta_{m_{1},-1,-1}^{7}, \ldots, \Delta_{M_{1},-2,-2}^{7}, \ldots, \Delta_{M_{1},-1,-1}^{7}\right\}^{\mathrm{T}}(\Delta=A, B, C) \text {, } \\
& \lambda_{m_{1}}^{a}=\frac{m_{1} \pi}{a}, \\
& \lambda_{n_{1}}^{b}=\frac{n_{1} \pi}{b}, \\
& \lambda_{l_{1}}^{h}=\frac{l_{1} \pi}{h} \text {. }
\end{aligned}
$$


2.4. Solution Procedure. With the aim of extending Ritz method to the partial differential operation of the unknown coefficients about above displacement functions, the kinetic energy as described below can be obtained by the way of replacing equations (9a)-(9c) with the kinetic energy $(T)$ function:

$$
\mathbf{T}=\frac{1}{2} \int_{0}^{h} \int_{0}^{b} \int_{0}^{a} \rho \omega^{2}\left[\left(\left(\mathbf{U}^{\Omega}+\sum_{q=1}^{6} \mathbf{U}_{q}^{\mathrm{S}}\right) \mathbf{A}_{m n q}\right)^{2}+\left(\left(\mathbf{V}^{\Omega}+\sum_{q=1}^{6} \mathbf{V}_{q}^{\mathrm{S}}\right) \mathbf{B}_{m n q}\right)^{2}+\left(\left(\mathbf{W}^{\Omega}+\sum_{q=1}^{6} \mathbf{W}_{q}^{\mathrm{S}}\right) \mathbf{C}_{m n q}\right)^{2}\right] d x d y d z
$$

In addition, by substituting equations (9a)-(9c) into an expression about strain energy, the following kinetic energy can be obtained:

$$
\begin{aligned}
& \mathbf{U}=\frac{1}{2} \int_{0}^{h} \int_{0}^{b} \int_{0}^{a}+\left\{C_{11}\left(\left(\frac{\partial \mathbf{U}^{\Omega}}{\partial x}+\sum_{q=1}^{6} \frac{\partial \mathbf{U}_{q}^{S}}{\partial x}\right) \mathbf{A}_{m n q}\right)^{2}+C_{22}\left(\left(\frac{\partial \mathbf{V}^{\Omega}}{\partial y}+\sum_{q=1}^{6} \frac{\partial \mathbf{V}_{q}^{S}}{\partial y}\right) \mathbf{B}_{m n q}\right)^{2}+C_{33}\left(\left(\frac{\partial \mathbf{W}^{\Omega}}{\partial z}+\sum_{q=1}^{6} \frac{\partial \mathbf{W}_{q}^{S}}{\partial z}\right)^{\mathbf{C}_{m n q}}\right)^{2}\right. \\
& +2 C_{12}\left(\frac{\partial \mathbf{U}^{\Omega}}{\partial x}+\sum_{q=1}^{6} \frac{\partial \mathbf{U}_{q}^{S}}{\partial x}\right) \mathbf{A}_{m n q}\left(\frac{\partial \mathbf{V}^{\Omega}}{\partial y}+\sum_{q=1}^{6} \frac{\partial \mathbf{V}_{q}^{S}}{\partial y}\right) \mathbf{B}_{m n q}+2 C_{12}\left(\frac{\partial \mathbf{U}^{\Omega}}{\partial x}+\sum_{q=1}^{6} \frac{\partial \mathbf{U}_{q}^{S}}{\partial x}\right) \mathbf{A}_{m n q}\left(\frac{\partial \mathbf{W}^{\Omega}}{\partial z}+\sum_{q=1}^{6} \frac{\partial \mathbf{W}_{q}^{S}}{\partial z}\right) \mathbf{C}_{m n q} \\
& +2 C_{12}\left(\frac{\partial \mathbf{V}^{\Omega}}{\partial y}+\sum_{q=1}^{6} \frac{\partial \mathbf{V}_{q}^{S}}{\partial y}\right) \mathbf{B}_{m n q}\left(\frac{\partial \mathbf{W}^{\Omega}}{\partial z}+\sum_{q=1}^{6} \frac{\partial \mathbf{W}_{q}^{S}}{\partial z}\right) \mathbf{C}_{m n q}+C_{66}\left(\left(\frac{\partial \mathbf{U}^{\Omega}}{\partial y}+\sum_{q=1}^{6} \frac{\partial \mathbf{U}_{q}^{S}}{\partial y}\right) \mathbf{A}_{m n q}\right)^{2}+C_{55}\left(\left(\frac{\partial \mathbf{U}^{\Omega}}{\partial z}+\sum_{q=1}^{6} \frac{\partial \mathbf{U}_{q}^{S}}{\partial z}\right)^{\mathbf{A}_{m n q}}\right)^{2} \\
& +C_{66}\left(\left(\frac{\partial \mathbf{V}^{\Omega}}{\partial x}+\sum_{q=1}^{6} \frac{\partial \mathbf{V}_{q}^{S}}{\partial x}\right) \mathbf{B}_{m n q}\right)^{2}+C_{44}\left(\left(\frac{\partial \mathbf{V}^{\Omega}}{\partial z}+\sum_{q=1}^{6} \frac{\partial \mathbf{V}_{q}^{S}}{\partial z}\right) \mathbf{B}_{m n q}\right)^{2}+C_{55}\left(\left(\frac{\partial \mathbf{W}^{\Omega}}{\partial x}+\sum_{q=1}^{6} \frac{\partial \mathbf{W}_{q}^{S}}{\partial x}\right)_{m n q}\right)^{2} \\
& +C_{55}\left(\left(\frac{\partial \mathbf{W}^{\Omega}}{\partial y}+\sum_{q=1}^{6} \frac{\partial \mathbf{W}_{q}^{S}}{\partial y}\right) \mathbf{C}_{m n q}\right)^{2}+2 C_{66}\left(\frac{\partial \mathbf{U}^{\Omega}}{\partial y}+\sum_{q=1}^{6} \frac{\partial \mathbf{U}_{q}^{S}}{\partial y}\right) \mathbf{A}_{m n q}\left(\frac{\partial \mathbf{V}^{\Omega}}{\partial x}+\sum_{q=1}^{6} \frac{\partial \mathbf{V}_{q}^{S}}{\partial x}\right) \mathbf{B}_{m n q} \\
& \left.+2 C_{55}\left(\frac{\partial \mathbf{U}^{\Omega}}{\partial z}+\sum_{q=1}^{6} \frac{\partial \mathbf{U}_{q}^{S}}{\partial z}\right) \mathbf{A}_{m n q}\left(\frac{\partial \mathbf{W}^{\Omega}}{\partial x}+\sum_{q=1}^{6} \frac{\partial \mathbf{W}_{q}^{S}}{\partial x}\right) \mathbf{C}_{m n q}+2 C_{55}\left(\frac{\partial \mathbf{V}^{\Omega}}{\partial z}+\sum_{q=1}^{6} \frac{\partial \mathbf{V}_{q}^{S}}{\partial z}\right) \mathbf{B}_{m n q}\left(\frac{\partial \mathbf{W}^{\Omega}}{\partial y}+\sum_{q=1}^{6} \frac{\partial \mathbf{W}_{q}^{S}}{\partial y}\right) \mathbf{C}_{m n q}\right\} d x d y d z .
\end{aligned}
$$

Substituting equation (7) for equations (9a)-(9c) under the same processing form can be obtained:

$$
\begin{aligned}
\mathbf{U}_{\mathbf{s p}}= & \frac{1}{2} \int_{0}^{h} \int_{0}^{b}\left\{k_{x 0}^{u}\left(\left(\mathbf{U}^{\Omega}(0, y, z)+\sum_{q=1}^{6} \mathbf{U}_{q}^{\mathrm{S}}(0, y, z)\right) \mathbf{A}_{m n q}\right)^{2}+k_{x 1}^{u}\left(\left(\mathbf{U}^{\Omega}(a, y, z)+\sum_{q=1}^{6} \mathbf{U}_{q}^{\mathrm{S}}(a, y, z)\right) \mathbf{A}_{m n q}\right)^{2}\right. \\
& +k_{x 0}^{v}\left(\left(\mathbf{V}^{\Omega}(0, y, z)+\sum_{q=1}^{6} \mathbf{V}_{q}^{\mathrm{S}}(0, y, z)\right) \mathbf{B}_{m n q}\right)^{2}+k_{x 1}^{v}\left(\left(\mathbf{V}^{\Omega}(a, y, z)+\sum_{q=1}^{6} \mathbf{V}_{q}^{\mathrm{S}}(a, y, z)\right) \mathbf{B}_{m n q}\right)^{2} \\
& \left.+k_{x 0}^{w}\left(\left(\mathbf{W}^{\Omega}(0, y, z)+\sum_{q=1}^{6} \mathbf{W}_{q}^{\mathrm{S}}(0, y, z)\right) \mathbf{C}_{m n q}\right)^{2}+k_{x 1}^{w}\left(\left(\mathbf{W}^{\Omega}(a, y, z)+\sum_{q=1}^{6} \mathbf{W}_{q}^{\mathrm{S}}(a, y, z)\right) \mathbf{C}_{m n q}\right)^{2}\right\} d y d z \\
& +\frac{1}{2} \int_{0}^{h} \int_{0}^{a}\left\{k_{y 0}^{u}\left(\left(\mathbf{U}^{\Omega}(x, 0, z)+\sum_{q=1}^{6} \mathbf{U}_{q}^{\mathrm{S}}(x, 0, z)\right)^{2} \mathbf{A}_{m n q}\right)^{2}+k_{y 1}^{u}\left(\left(\mathbf{U}^{\Omega}(x, b, z)+\sum_{q=1}^{6} \mathbf{U}_{q}^{\mathrm{S}}(x, b, z)\right) \mathbf{A}_{m n q}\right)^{2}\right. \\
& +k_{y 0}^{v}\left(\left(\mathbf{V}^{\Omega}(x, 0, z)+\sum_{q=1}^{6} \mathbf{V}_{q}^{\mathrm{S}}(x, 0, z)\right) \mathbf{B}_{m n q}\right)^{2}+k_{y 1}^{v}\left(\left(\mathbf{V}^{\Omega}(x, b, z)+\sum_{q=1}^{6} \mathbf{V}_{q}^{\mathrm{S}}(x, b, z)\right) \mathbf{B}_{m n q}\right)^{2} \\
& \left.+k_{y 0}^{w}\left(\left(\mathbf{W}^{\Omega}(x, 0, z)+\sum_{q=1}^{6} \mathbf{W}_{q}^{\mathrm{S}}(x, 0, z)\right) \mathbf{C}_{m n q}\right)^{2}+k_{y 1}^{w}\left(\left(\mathbf{W}^{\Omega}(x, b, z)+\sum_{q=1}^{6} \mathbf{W}_{q}^{\mathrm{S}}(x, b, z)\right) \mathbf{C}_{m n q}\right)^{2}\right\} d x d z .
\end{aligned}
$$


TABLE 1: Convergence of frequency parameters $\Omega$ of an orthotropic plate with different truncated numbers.

\begin{tabular}{|c|c|c|c|c|c|c|c|c|}
\hline \multirow{2}{*}{$M * N * Q$} & \multicolumn{8}{|c|}{ Mode number } \\
\hline & 1 & 2 & 3 & 4 & 5 & 6 & 7 & 8 \\
\hline $4 * 4 * 4$ & 0.04764 & 0.10357 & 0.11900 & 0.16108 & 0.16108 & 0.16967 & 0.19038 & 0.21697 \\
\hline $5 * 5 * 5$ & 0.04745 & 0.10333 & 0.11883 & 0.16108 & 0.16108 & 0.16944 & 0.18950 & 0.21697 \\
\hline $6 * 6 * 6$ & 0.04745 & 0.10333 & 0.11883 & 0.16108 & 0.16108 & 0.16944 & 0.18904 & 0.21697 \\
\hline $7 * 7 * 7$ & 0.04742 & 0.10330 & 0.11880 & 0.16108 & 0.16108 & 0.16942 & 0.18894 & 0.21697 \\
\hline $8 * 8 * 8$ & 0.04742 & 0.10330 & 0.11880 & 0.16108 & 0.16108 & 0.16942 & 0.18887 & 0.21697 \\
\hline $9 * 9 * 9$ & 0.04742 & 0.10329 & 0.11880 & 0.16108 & 0.16108 & 0.16941 & 0.18885 & 0.21697 \\
\hline $10 * 10 * 10$ & 0.04742 & 0.10329 & 0.11880 & 0.16108 & 0.16108 & 0.16941 & 0.18883 & 0.21697 \\
\hline DQM & 0.04742 & 0.10329 & 0.11880 & 0.16109 & 0.16109 & 0.16941 & 0.18876 & 0.21697 \\
\hline $4 * 4 * 4$ & 0.33231 & 0.48323 & 0.48323 & 0.65021 & 0.65043 & 0.65665 & 0.89812 & 0.96645 \\
\hline $5 * 5 * 5$ & 0.33203 & 0.48323 & 0.48323 & 0.64980 & 0.65043 & 0.65618 & 0.89750 & 0.96645 \\
\hline $6 * 6 * 6$ & 0.33203 & 0.48323 & 0.48323 & 0.64979 & 0.65043 & 0.65618 & 0.89750 & 0.96645 \\
\hline $7 * 7 * 7$ & 0.33200 & 0.48323 & 0.48323 & 0.64974 & 0.65043 & 0.65611 & 0.89741 & 0.96645 \\
\hline $8 * 8 * 8$ & 0.33200 & 0.48323 & 0.48323 & 0.64974 & 0.65043 & 0.65611 & 0.89741 & 0.96645 \\
\hline $9 * 9 * 9$ & 0.33199 & 0.48323 & 0.48323 & 0.64973 & 0.65043 & 0.65610 & 0.89739 & 0.96645 \\
\hline $10 * 10 * 10$ & 0.33199 & 0.48323 & 0.48323 & 0.64973 & 0.65043 & 0.65610 & 0.89739 & 0.96645 \\
\hline DQM & 0.33200 & 0.48327 & 0.48327 & 0.64974 & 0.65043 & 0.65612 & 0.89742 & 0.96654 \\
\hline $4 * 4 * 4$ & 0.70391 & 0.80538 & 0.80538 & 1.08235 & 1.24394 & 1.30523 & 1.49227 & 1.61076 \\
\hline $5 * 5 * 5$ & 0.70344 & 0.80538 & 0.80538 & 1.08235 & 1.24259 & 1.30446 & 1.49227 & 1.61076 \\
\hline $6 * 6 * 6$ & 0.70343 & 0.80538 & 0.80538 & 1.08235 & 1.24257 & 1.30444 & 1.49227 & 1.61076 \\
\hline $7 * 7 * 7$ & 0.70337 & 0.80538 & 0.80538 & 1.08234 & 1.24237 & 1.30434 & 1.49227 & 1.61076 \\
\hline $8 * 8 * 8$ & 0.70337 & 0.80538 & 0.80538 & 1.08234 & 1.24237 & 1.30433 & 1.49227 & 1.61076 \\
\hline $9 * 9 * 9$ & 0.70336 & 0.80538 & 0.80538 & 1.08234 & 1.24232 & 1.30431 & 1.49227 & 1.61076 \\
\hline $10 * 10 * 10$ & 0.70336 & 0.80538 & 0.80538 & 1.08234 & 1.24232 & 1.30431 & 1.49227 & 1.61076 \\
\hline DQM & 0.70338 & 0.80545 & 0.80545 & 1.08240 & 1.24230 & 1.30430 & 1.49230 & 1.61090 \\
\hline
\end{tabular}

TABLE 2: Comparison of the frequency parameters $\Omega$ of an orthotropic plate elastically restrained in the $x$ direction.

\begin{tabular}{|c|c|c|c|c|c|c|c|c|}
\hline \multirow{2}{*}{$k_{u}$} & \multicolumn{8}{|c|}{ Mode number } \\
\hline & 1 & 2 & 3 & 4 & 5 & 6 & 7 & 8 \\
\hline $10^{2}$ & 0.18702 & 0.29162 & 0.29497 & 0.62104 & 0.74459 & 0.78604 & 0.80941 & 0.84366 \\
\hline $10^{4}$ & 0.18702 & 0.29162 & 0.29497 & 0.62104 & 0.74459 & 0.78604 & 0.80941 & 0.84366 \\
\hline $10^{6}$ & 0.18702 & 0.29162 & 0.29497 & 0.62105 & 0.74459 & 0.78604 & 0.80941 & 0.84366 \\
\hline $10^{8}$ & 0.18705 & 0.29166 & 0.29497 & 0.62112 & 0.74486 & 0.78620 & 0.80942 & 0.84369 \\
\hline $10^{10}$ & 0.19001 & 0.29513 & 0.29519 & 0.62816 & 0.77115 & 0.80077 & 0.80960 & 0.84620 \\
\hline $10^{12}$ & 0.22782 & 0.29815 & 0.33392 & 0.75364 & 0.81725 & 0.88758 & 1.02990 & 1.09273 \\
\hline $10^{14}$ & 0.23366 & 0.29893 & 0.33899 & 0.77624 & 0.82308 & 0.89642 & 1.06101 & 1.09704 \\
\hline $10^{16}$ & 0.23372 & 0.29894 & 0.33904 & 0.77648 & 0.82317 & 0.89653 & 1.06135 & 1.09709 \\
\hline $10^{18}$ & 0.23372 & 0.29894 & 0.33904 & 0.77648 & 0.82318 & 0.89653 & 1.06135 & 1.09709 \\
\hline
\end{tabular}

TABLE 3: Comparison of the frequency parameters $\Omega$ of an orthotropic plate elastically restrained in the $y$ direction.

\begin{tabular}{|c|c|c|c|c|c|c|c|c|}
\hline \multirow{2}{*}{$k_{u}$} & \multicolumn{8}{|c|}{ Mode number } \\
\hline & 1 & 2 & 3 & 4 & 5 & 6 & 7 & 8 \\
\hline $10^{2}$ & 0.18702 & 0.29162 & 0.29497 & 0.62104 & 0.74459 & 0.78604 & 0.80941 & 0.84366 \\
\hline $10^{4}$ & 0.18702 & 0.29162 & 0.29497 & 0.62104 & 0.74459 & 0.78604 & 0.80941 & 0.84366 \\
\hline $10^{6}$ & 0.18702 & 0.29163 & 0.29497 & 0.62104 & 0.74459 & 0.78604 & 0.80942 & 0.84366 \\
\hline $10^{8}$ & 0.18702 & 0.29259 & 0.29510 & 0.62105 & 0.74459 & 0.78616 & 0.80951 & 0.84367 \\
\hline $10^{10}$ & 0.18702 & 0.30698 & 0.36985 & 0.62137 & 0.74470 & 0.79840 & 0.81790 & 0.84449 \\
\hline $10^{12}$ & 0.18705 & 0.43816 & 0.62392 & 0.65914 & 0.74518 & 0.85657 & 0.90849 & 1.07874 \\
\hline $10^{14}$ & 0.18706 & 0.45980 & 0.62422 & 0.66717 & 0.74521 & 0.86111 & 0.92234 & 1.10855 \\
\hline $10^{16}$ & 0.18706 & 0.46010 & 0.62423 & 0.66725 & 0.74521 & 0.86121 & 0.92254 & 1.10886 \\
\hline $10^{18}$ & 0.18706 & 0.46010 & 0.62423 & 0.66725 & 0.74521 & 0.86121 & 0.92254 & 1.10886 \\
\hline
\end{tabular}


TABLE 4: Comparison of the frequency parameters $\Omega$ of an orthotropic plate elastically restrained in the $z$ direction.

\begin{tabular}{|c|c|c|c|c|c|c|c|c|}
\hline \multirow{2}{*}{$k_{u}$} & \multicolumn{8}{|c|}{ Mode number } \\
\hline & 1 & 2 & 3 & 4 & 5 & 6 & 7 & 8 \\
\hline $10^{2}$ & 0.18702 & 0.29162 & 0.29497 & 0.62104 & 0.74459 & 0.78604 & 0.80941 & 0.84366 \\
\hline $10^{4}$ & 0.18702 & 0.29162 & 0.29497 & 0.62104 & 0.74459 & 0.78604 & 0.80941 & 0.84366 \\
\hline $10^{6}$ & 0.18703 & 0.29162 & 0.29498 & 0.62105 & 0.74459 & 0.78604 & 0.80942 & 0.84366 \\
\hline $10^{8}$ & 0.18875 & 0.29162 & 0.29562 & 0.62138 & 0.74459 & 0.78604 & 0.80959 & 0.84397 \\
\hline $10^{10}$ & 0.29162 & 0.29912 & 0.34712 & 0.65655 & 0.74459 & 0.78604 & 0.82568 & 0.87358 \\
\hline $10^{12}$ & 0.29163 & 0.48109 & 0.53466 & 0.74461 & 0.78605 & 0.94237 & 1.05215 & 1.06005 \\
\hline $10^{14}$ & 0.29163 & 0.48454 & 0.54369 & 0.74461 & 0.78606 & 0.95355 & 1.05293 & 1.07903 \\
\hline $10^{16}$ & 0.29163 & 0.48458 & 0.54379 & 0.74461 & 0.78606 & 0.95368 & 1.05294 & 1.07922 \\
\hline $10^{18}$ & 0.29163 & 0.48458 & 0.54379 & 0.74461 & 0.78606 & 0.95368 & 1.05294 & 1.07922 \\
\hline
\end{tabular}

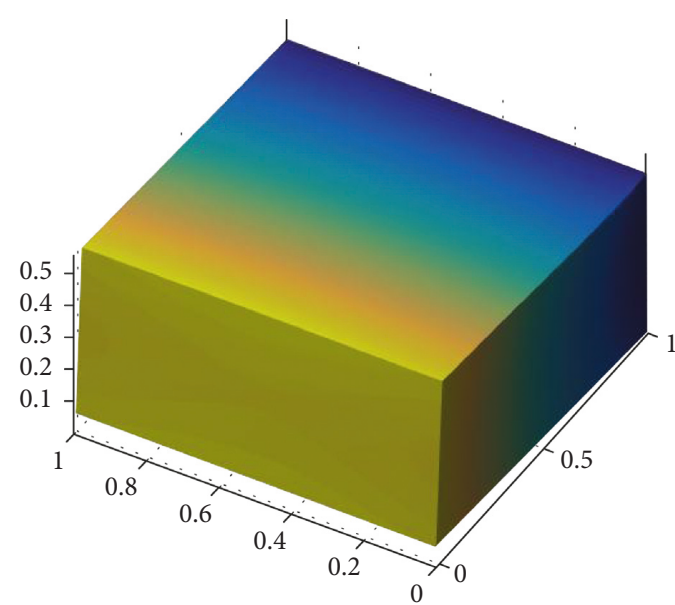

(a)

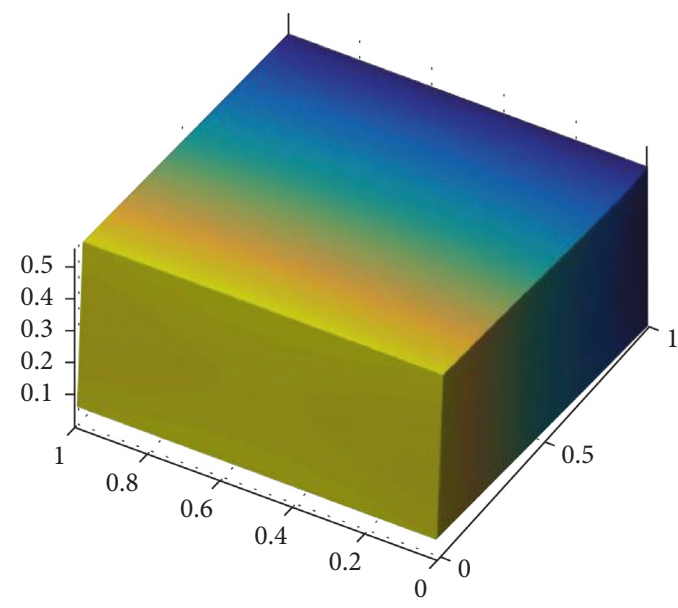

(c)

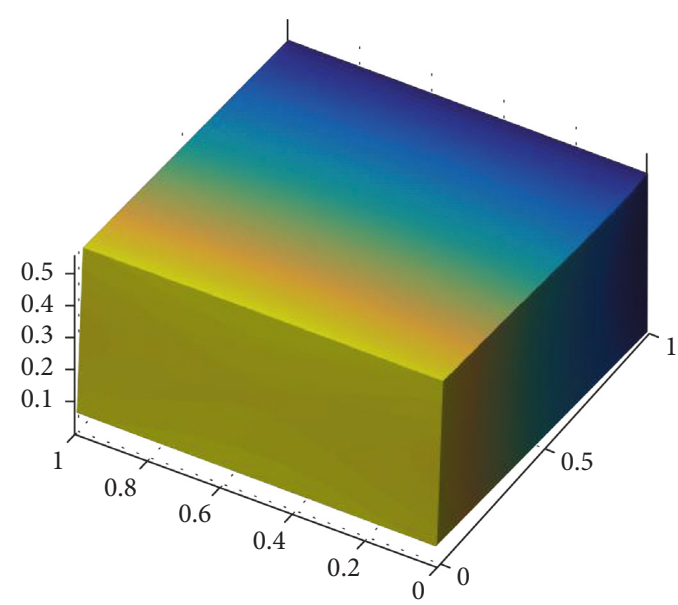

(b)

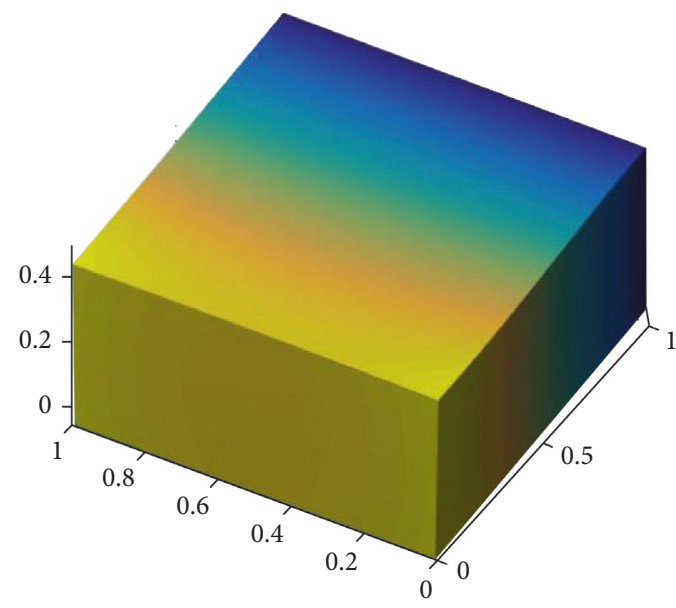

(d)

Figure 2: Mode shapes of the elastically supported plate elastically restrained in the $x$ direction: (a) $k_{u}=10^{2}$; (b) $k_{u}=10^{6}$; (c) $k_{u}=10^{10}$; (d) $k_{u}=10^{16}$.

The Lagrangian equation [31] $\mathbf{L}$ of the vibration of the thick orthotropic plate structure can be obtained in the light of the above expression, and the equation is expressed in the following manner:

$$
\mathbf{L}=\mathbf{U}+\mathbf{U}_{\text {sp }}-\mathbf{T} .
$$

In addition, equations (11)-(13) are substituted into equation (14), and then the Lagrangian equations are partially deduced to zero according to the sealed Fourier coefficient vector, and its expression is as follows:

$$
\frac{\partial \mathbf{L}}{\partial \mathbf{A}_{m n q}}=\frac{\partial \mathbf{L}}{\partial \mathbf{B}_{m n q}}=\frac{\partial \mathbf{L}}{\partial \mathbf{C}_{m n q}}=\mathbf{0}
$$




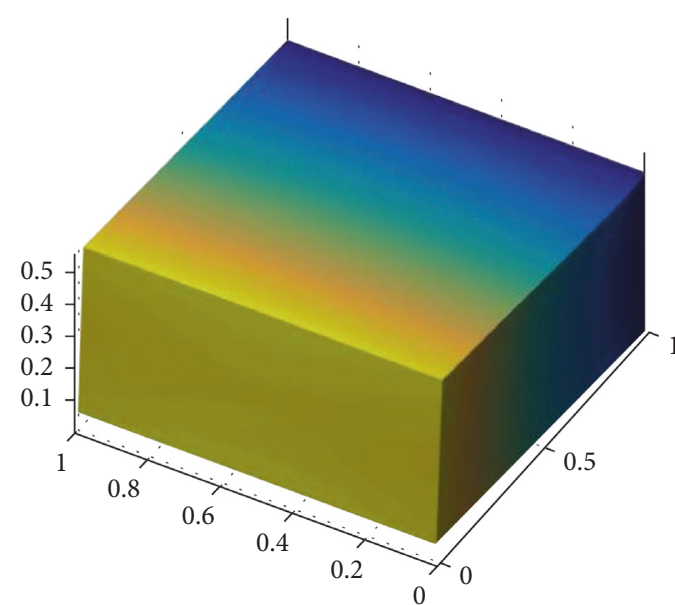

(a)

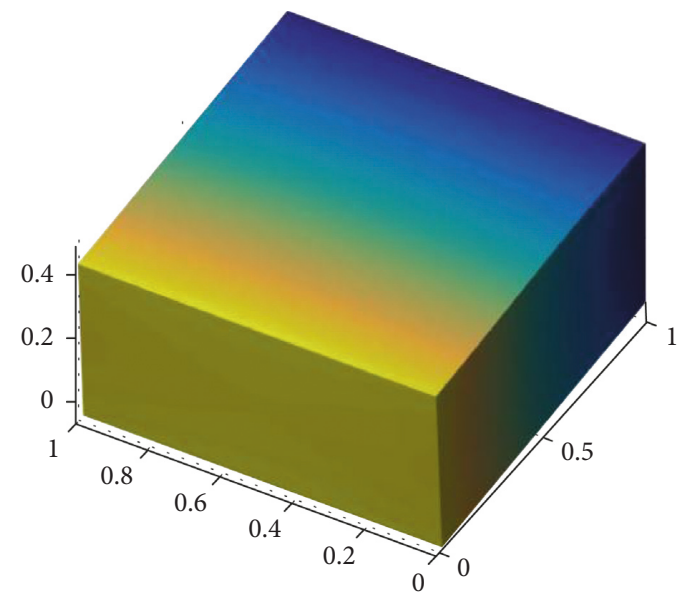

(c)

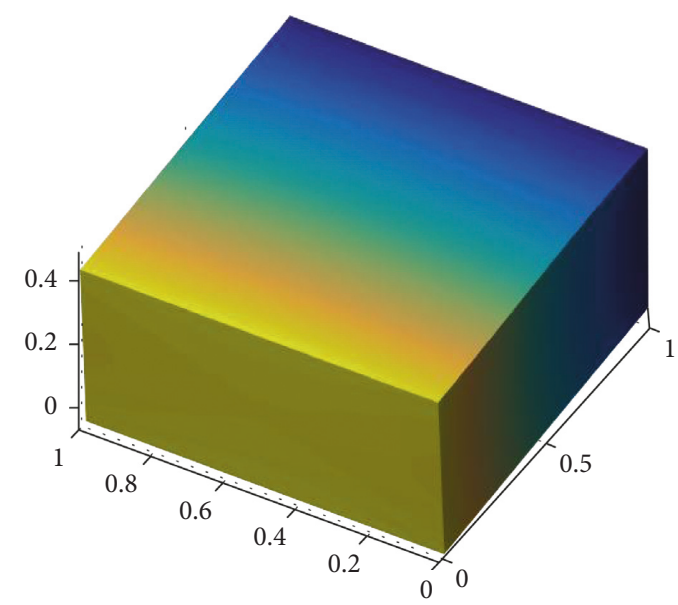

(b)

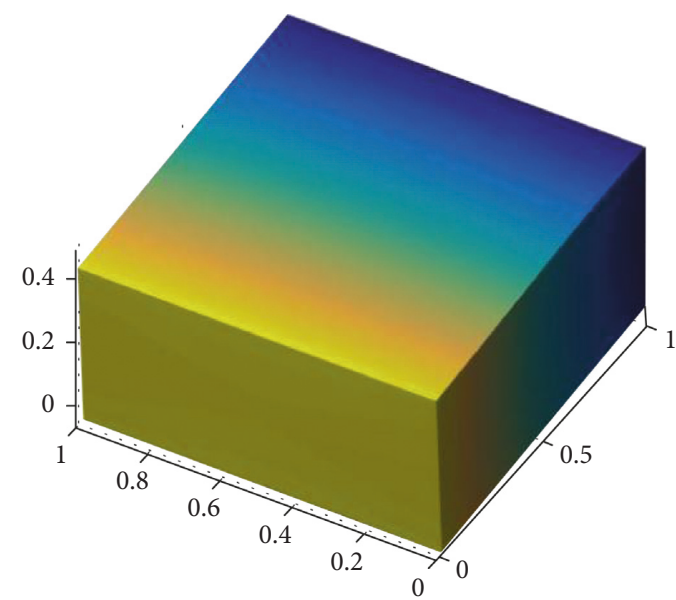

(d)

Figure 3: Mode shapes of the elastically supported plate elastically restrained in the $y$ direction: (a) $k_{v}=10^{2}$; (b) $k_{v}=10^{6}$; (c) $k_{v}=10^{10}$; (d) $k_{v}=10^{16}$.

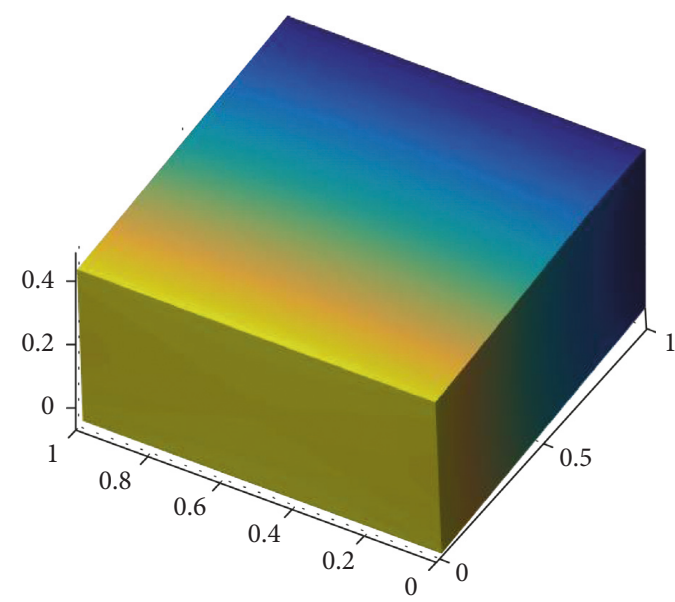

(a)

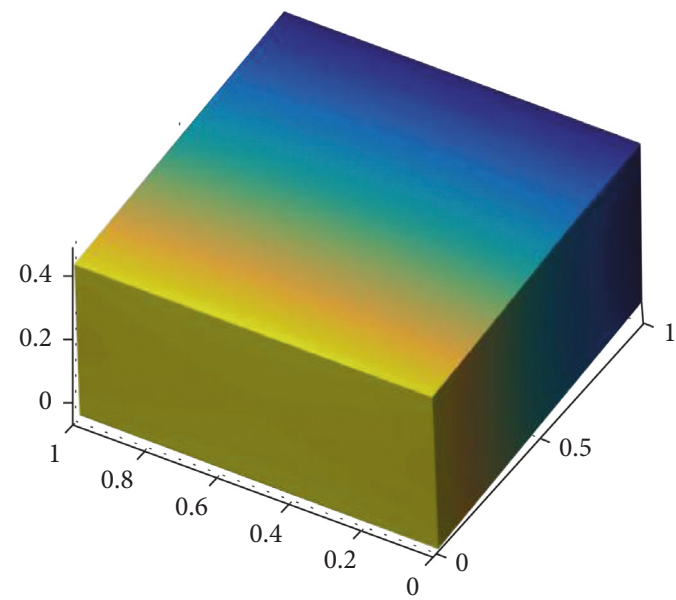

(b)

Figure 4: Continued. 


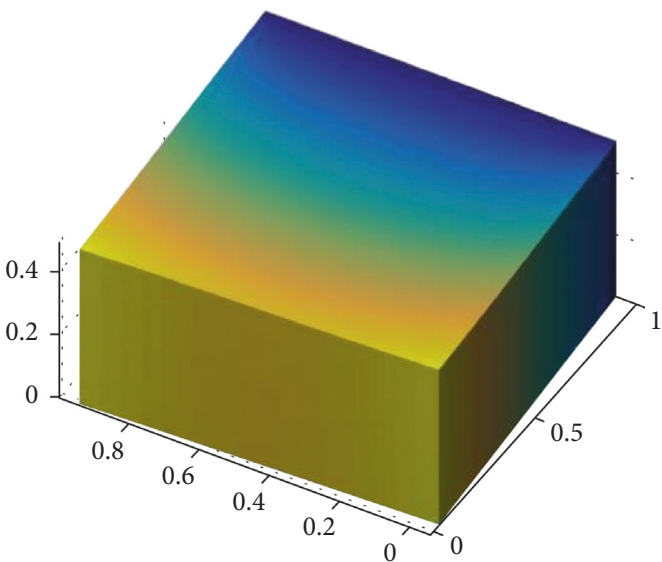

(c)

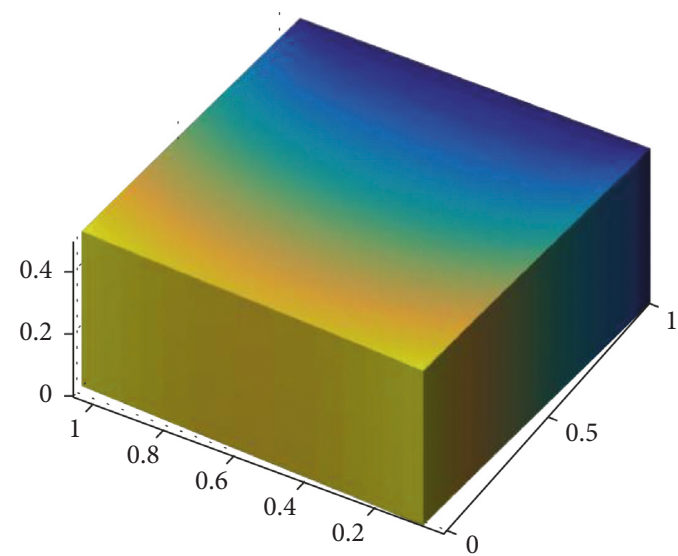

(d)

Figure 4: Mode shapes of the elastically supported plate elastically restrained in the $z$ direction: (a) $k_{v}=10^{2}$; (b) $k_{v}=10^{6}$; (c) $k_{v}=10^{10}$; (d) $k_{v}=10^{16}$.

TABle 5: Values of spring stiffness for three types of classical boundary conditions and four kinds of elastic constraint.

\begin{tabular}{lccc}
\hline Boundary condition & \multicolumn{3}{c}{ Spring stiffness } \\
& $k_{u}$ & $k_{v}$ & $k_{w}$ \\
\hline Free edge (F) & 0 & 0 & 0 \\
Simply supported edge (S) & 0 & $10^{16}$ & $10^{16}$ \\
Clamped edge (C) & $10^{16}$ & $10^{16}$ & $10^{16}$ \\
Elastic constraint 1 (E1) & $10^{10}$ & $10^{16}$ & $10^{16}$ \\
Elastic constraint 2 (E2) & $10^{16}$ & $10^{10}$ & $10^{16}$ \\
Elastic constraint 3 (E3) & $10^{16}$ & $10^{16}$ & $10^{10}$ \\
Elastic constraint 4 (E4) & $10^{10}$ & $10^{10}$ & $10^{10}$ \\
\hline
\end{tabular}

TABle 6: Comparison of frequency parameters of a thick plate with different boundary conditions.

\begin{tabular}{|c|c|c|c|c|c|c|c|c|c|c|c|c|}
\hline \multirow{3}{*}{ Mode } & \multicolumn{4}{|c|}{ СССС } & \multicolumn{4}{|c|}{ SCCC } & \multicolumn{4}{|c|}{ CSSS } \\
\hline & \multicolumn{2}{|c|}{0.3} & \multicolumn{2}{|c|}{0.5} & \multicolumn{2}{|c|}{0.3} & \multicolumn{2}{|c|}{0.5} & \multicolumn{2}{|c|}{0.3} & \multicolumn{2}{|c|}{0.5} \\
\hline & $\begin{array}{c}\text { Reference } \\
{[23]}\end{array}$ & Present & $\begin{array}{c}\text { Reference } \\
{[23]}\end{array}$ & Present & $\begin{array}{c}\text { Reference } \\
{[23]}\end{array}$ & Present & $\begin{array}{c}\text { Reference } \\
{[23]}\end{array}$ & Present & $\begin{array}{c}\text { Reference } \\
{[23]}\end{array}$ & Present & $\begin{array}{c}\text { Reference } \\
{[23]}\end{array}$ & Present \\
\hline 1 & 0.44253 & 0.44282 & 0.85276 & 0.85333 & 0.42055 & 0.42081 & 0.82740 & & 0.35129 & 0.35137 & 0.71542 & \\
\hline 2 & 0.71315 & 0.71373 & 1.30250 & 30365 & 0.66043 & 0.66037 & 80 & & 27 & 0.48323 & 45 & 0.80538 \\
\hline 3 & 0.74996 & 0.75068 & 1.36150 & 1.36148 & 0.69853 & 0.69887 & 1.29250 & 1.29318 & 0.58091 & 0.58088 & 0.96775 & 0.96771 \\
\hline 4 & 0.81676 & 0.81678 & 1.39330 & 1.39421 & 0.74541 & 0.74609 & 1.29710 & 1.29712 & 0.65229 & 0.65234 & 1.25290 & 1.25337 \\
\hline 5 & 0.96568 & 0.96659 & 1.70400 & & & 0.77816 & & & & 0.67164 & & 1.30682 \\
\hline 6 & 1.02230 & 1.02214 & 1.76040 & 1.76190 & 0.95912 & 0.95985 & 1.67530 & 1.67526 & 0.84199 & 0.84190 & 1.40000 & 1.39988 \\
\hline 7 & 1.04660 & 1.04768 & 1.79350 & 1.79348 & 1.03540 & 1.03605 & 1.75510 & 1.75623 & 0.90230 & 0.90245 & 1.61090 & 1.61076 \\
\hline 8 & 1.07660 & 1.07657 & 1.86190 & 1.86430 & 1.05620 & 1.05610 & 1.76000 & 1.75989 & 0.96654 & 0.96645 & 1.63620 & 1.63612 \\
\hline
\end{tabular}

TABle 7: Comparison of the natural frequencies for the isotropic plate.

\begin{tabular}{lccccc}
\hline Mode number & Present & Experimental & Error (\%) & FEM & Error (\%) \\
\hline 1 & 329.1 & 331.4 & 0.70 & 329.8 & 0.21 \\
2 & 802.3 & 811.5 & 1.15 & 806.6 & 0.54 \\
3 & 1538.9 & 1564.7 & 1.68 & 1552.8 & 0.90 \\
\hline
\end{tabular}




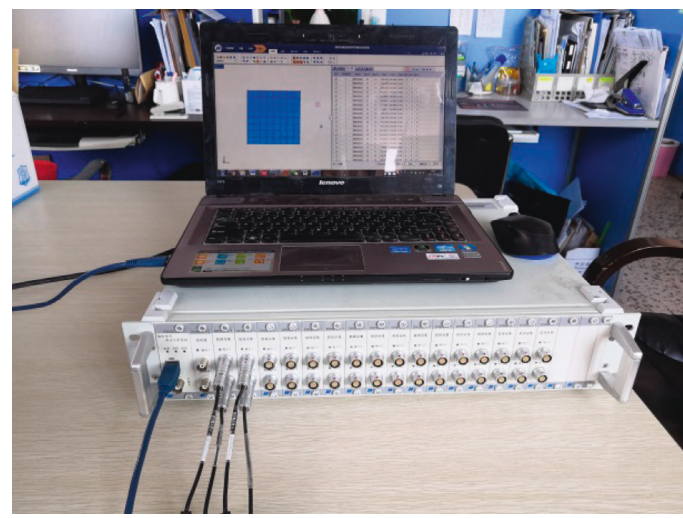

(a)

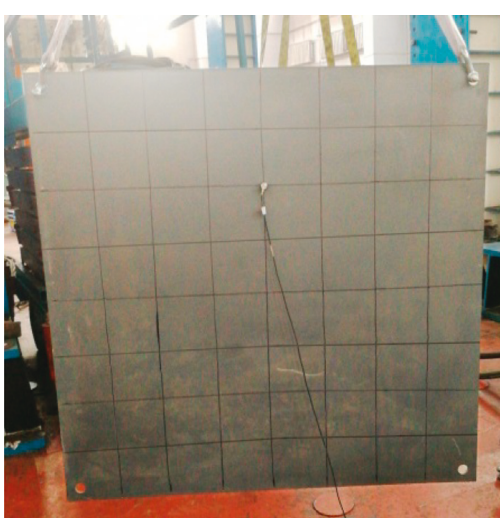

(b)

Figure 5: (a) Test system; (b) test model.

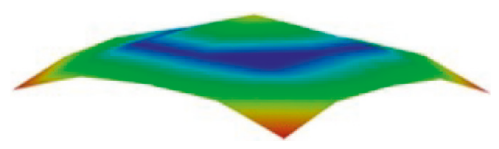

(a)

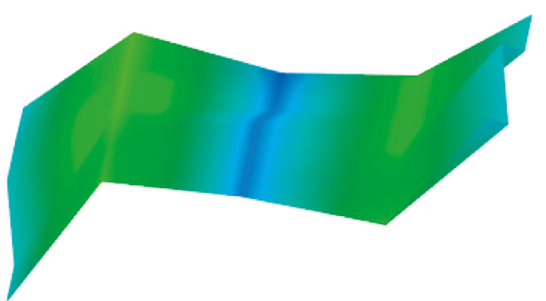

(b)

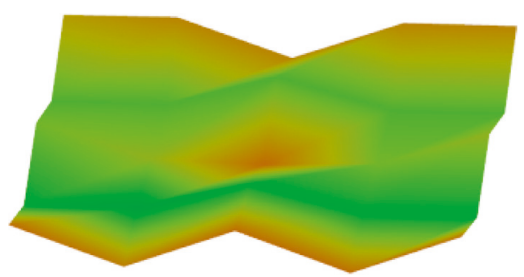

(c)

FIGURE 6: First three modes shapes of the experiment: (a) first mode shape; (b) second mode shape; (c) third mode shape.

TABLE 8: First eight frequency parameters $\Omega$ of an orthotropic plate with different boundary conditions $(h / a=0.3)$.

\begin{tabular}{|c|c|c|c|c|c|c|c|c|}
\hline \multirow{2}{*}{ Mode number } & \multicolumn{8}{|c|}{ Boundary conditions } \\
\hline & $\mathrm{CCCC}$ & SSSS & CFCF & SFSF & E1E1E1E1 & E2E2E2E2 & E3E3E3E3 & E4E4E4E4 \\
\hline 1 & 0.44282 & 0.33200 & 0.29858 & 0.19759 & 0.17039 & 0.16987 & 0.16384 & 0.15148 \\
\hline 2 & 0.71373 & 0.48323 & 0.32960 & 0.27621 & 0.39345 & 0.36694 & 0.30514 & 0.16813 \\
\hline 3 & 0.75068 & 0.48323 & 0.44653 & 0.38466 & 0.52665 & 0.52642 & 0.31104 & 0.16823 \\
\hline 4 & 0.81678 & 0.64974 & 0.50323 & 0.48326 & 0.66414 & 0.65042 & 0.47775 & 0.22784 \\
\hline 5 & 0.96659 & 0.65043 & 0.60714 & 0.49437 & 0.67670 & 0.67737 & 0.61966 & 0.22816 \\
\hline 6 & 1.02214 & 0.65611 & 0.65953 & 0.55985 & 0.73408 & 0.68260 & 0.62444 & 0.23910 \\
\hline 7 & 1.04768 & 0.89741 & 0.76750 & 0.62422 & 0.94386 & 0.71401 & 0.74591 & 0.35537 \\
\hline 8 & 1.07657 & 0.96645 & 0.81376 & 0.63107 & 0.96491 & 0.89351 & 0.77238 & 0.40865 \\
\hline
\end{tabular}

TABle 9: First eight frequency parameters $\Omega$ of an orthotropic plate with different boundary conditions $(h / a=0.6)$.

\begin{tabular}{|c|c|c|c|c|c|c|c|c|}
\hline \multirow{2}{*}{ Mode number } & \multicolumn{8}{|c|}{ Boundary conditions } \\
\hline & CCCC & SSSS & CFCF & SFSF & E1E1E1E1 & E2E2E2E2 & E3E3E3E3 & $\mathrm{E} 4 \mathrm{E} 4 \mathrm{E} 4 \mathrm{E} 4$ \\
\hline 1 & 1.05986 & 0.89741 & 0.68020 & 0.55575 & 0.34079 & 0.33975 & 0.33217 & 0.32414 \\
\hline 2 & 1.60031 & 0.96645 & 0.76402 & 0.72993 & 0.99500 & 0.89388 & 0.73078 & 0.33626 \\
\hline 3 & 1.63388 & 0.96645 & 0.89309 & 0.76929 & 1.05329 & 1.05285 & 0.76442 & 0.33647 \\
\hline 4 & 1.70799 & 1.29724 & 1.25588 & 0.96651 & 1.30164 & 1.36600 & 1.12105 & 0.44272 \\
\hline 5 & 2.04465 & 1.53683 & 1.33820 & 1.21143 & 1.32986 & 1.42322 & 1.40388 & 0.44290 \\
\hline 6 & 2.15175 & 1.58501 & 1.49164 & 1.25628 & 1.57023 & 1.51977 & 1.57675 & 0.47822 \\
\hline 7 & 2.15786 & 1.63841 & 1.52677 & 1.25813 & 1.63941 & 1.63627 & 1.61920 & 0.85949 \\
\hline 8 & 2.27251 & 1.88870 & 1.71551 & 1.31246 & 1.70046 & 1.66079 & 1.66279 & 1.03227 \\
\hline
\end{tabular}


TABLE 10: First eight frequency parameters $\Omega$ of an orthotropic plate with different boundary conditions $(h / a=1)$.

\begin{tabular}{|c|c|c|c|c|c|c|c|c|}
\hline \multirow{2}{*}{ Mode number } & \multicolumn{8}{|c|}{ Boundary conditions } \\
\hline & CCCC & SSSS & CFCF & SFSF & E1E1E1E1 & E2E2E2E2 & E3E3E3E3 & E4E4E4E4 \\
\hline 1 & 1.88150 & 1.61076 & 1.18445 & 1.05760 & 0.56798 & 0.56627 & 0.55563 & 0.54909 \\
\hline 2 & 2.72568 & 1.61076 & 1.36175 & 1.25628 & 1.37869 & 1.66037 & 1.30379 & 0.56044 \\
\hline 3 & 2.77687 & 1.68779 & 1.48851 & 1.28218 & 1.75549 & 1.71853 & 1.44804 & 0.56080 \\
\hline 4 & 2.78767 & 2.04273 & 2.31018 & 1.31711 & 1.81113 & 1.75476 & 1.99754 & 0.68732 \\
\hline 5 & 2.89328 & 2.13744 & 2.31299 & 1.61086 & 2.15870 & 2.27932 & 2.35262 & 0.68739 \\
\hline 6 & 3.37654 & 2.28641 & 2.33043 & 2.04281 & 2.22119 & 2.30082 & 2.37089 & 0.79706 \\
\hline 7 & 3.38005 & 2.55773 & 2.38591 & 2.05895 & 2.57591 & 2.38993 & 2.44314 & 1.47435 \\
\hline 8 & 3.38707 & 2.66766 & 2.40352 & 2.11317 & 2.62957 & 2.57678 & 2.52716 & 1.67874 \\
\hline
\end{tabular}

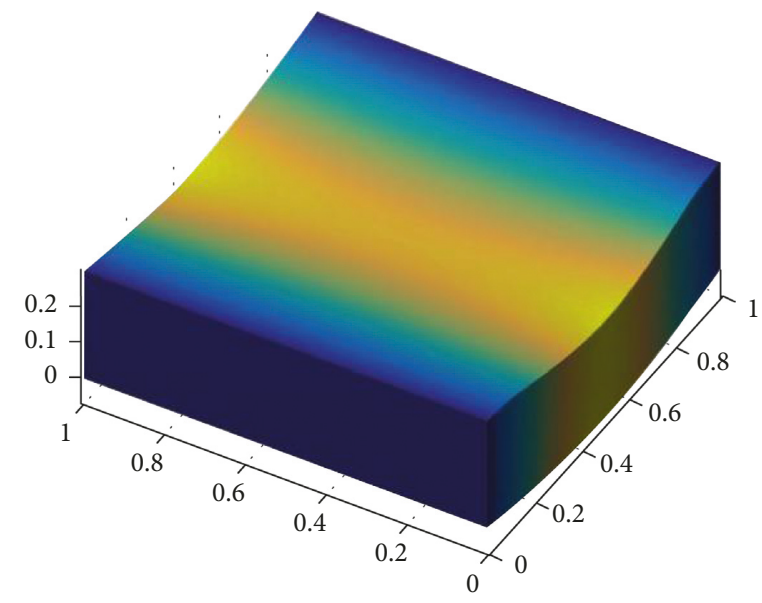

(a)

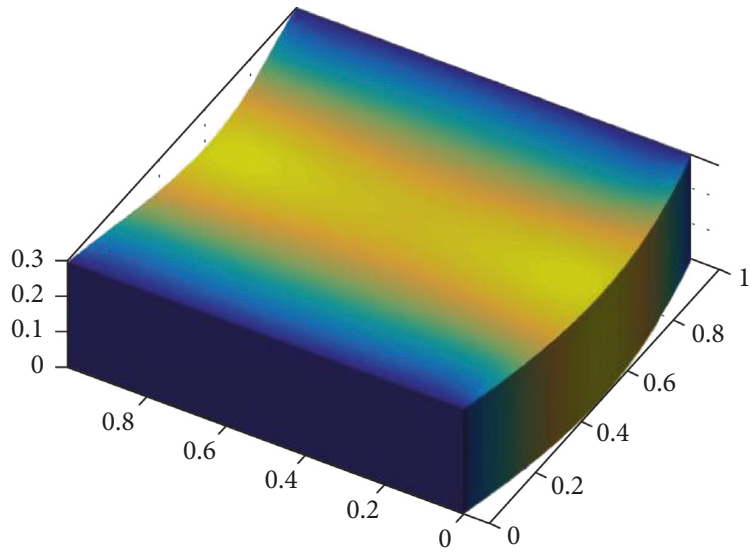

(c)

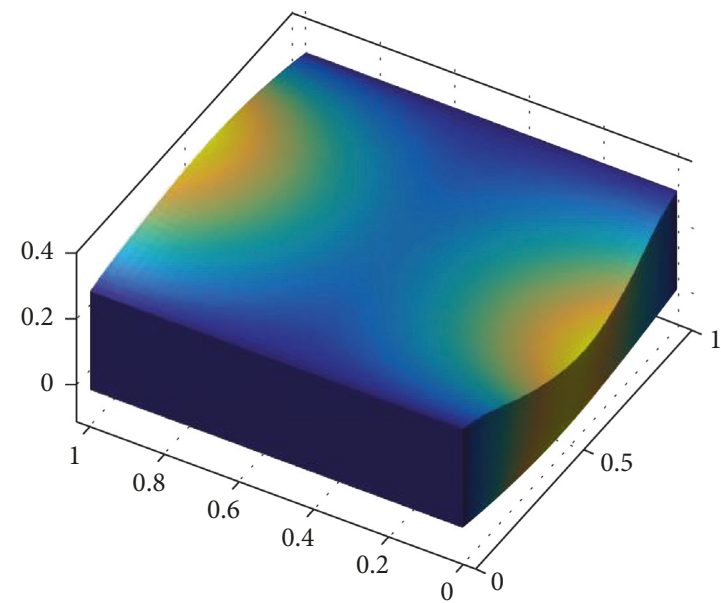

(b)

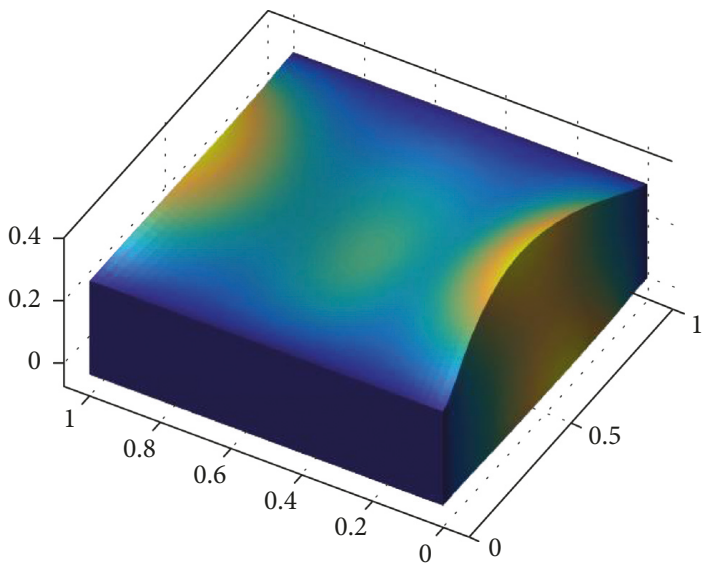

(d)

Figure 7: Continued. 


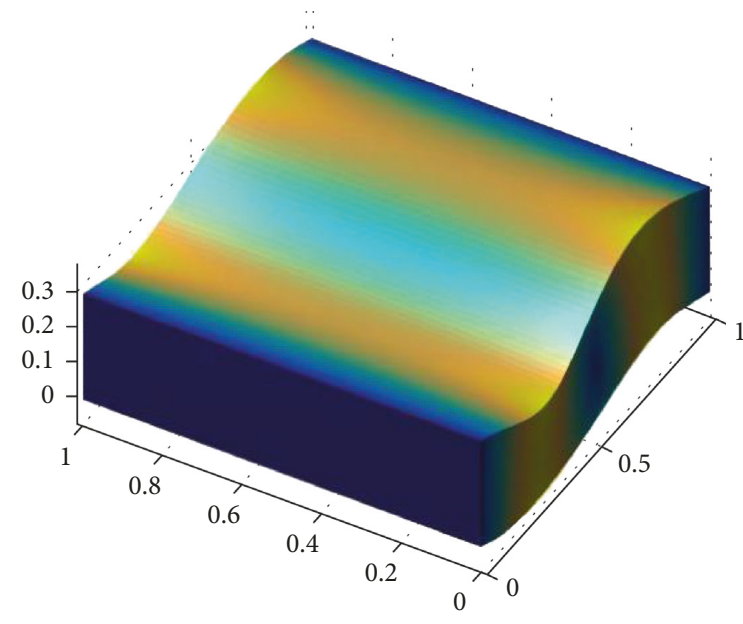

(e)

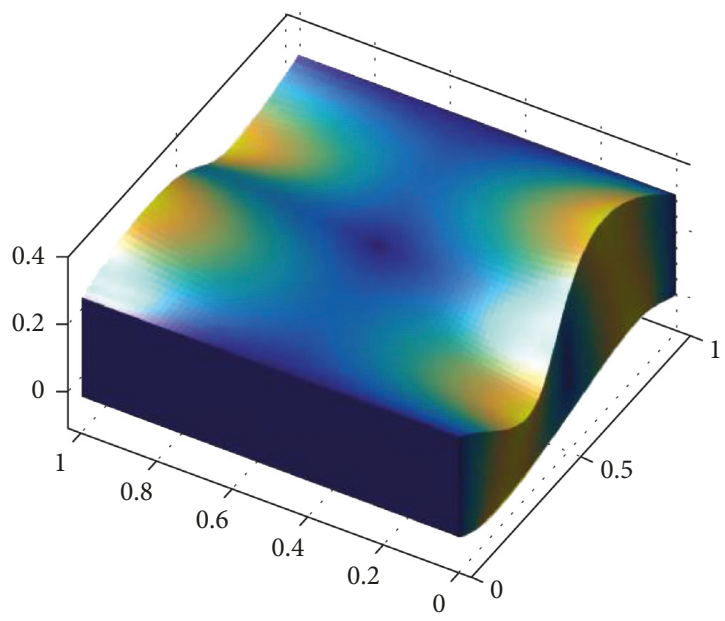

(f)

FIGURE 7: Mode shapes of orthotropic rectangular plates with the CFCF boundary condition: (a) 1st mode; (b) 2nd mode; (c) 3rd mode; (d) 4th mode; (e) 5th mode; (f) 6th mode.

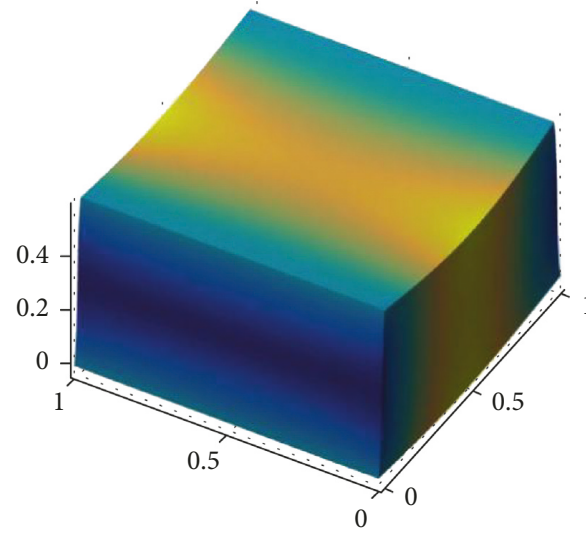

(a)

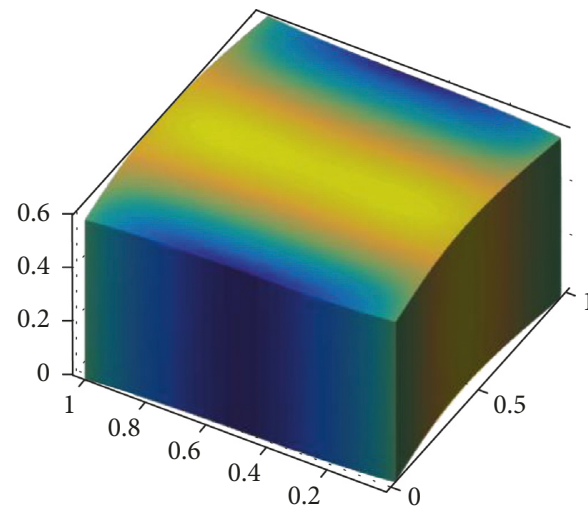

(c)

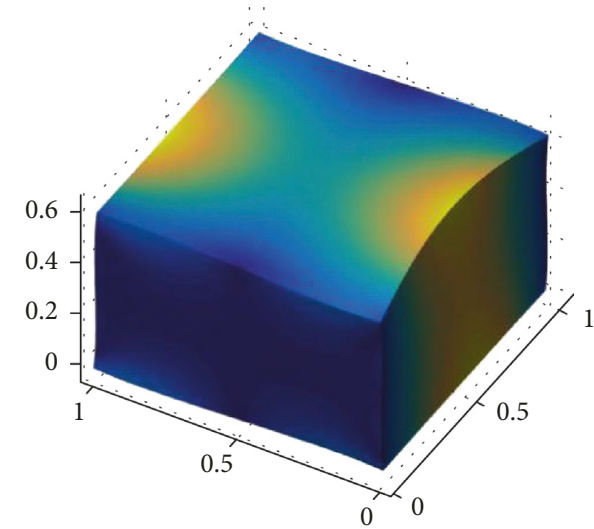

(b)

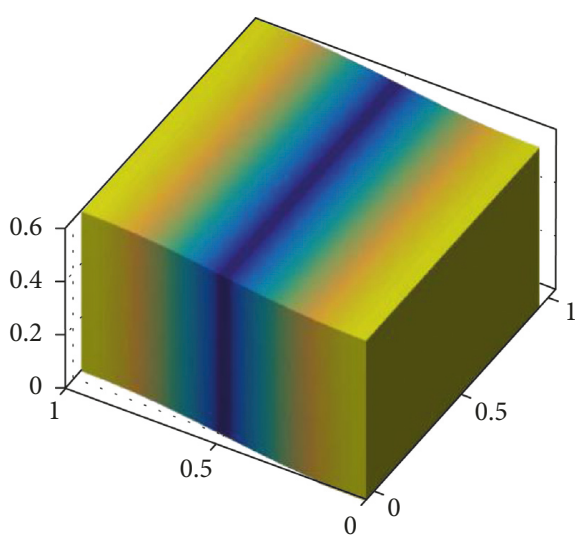

(d)

Figure 8: Continued. 


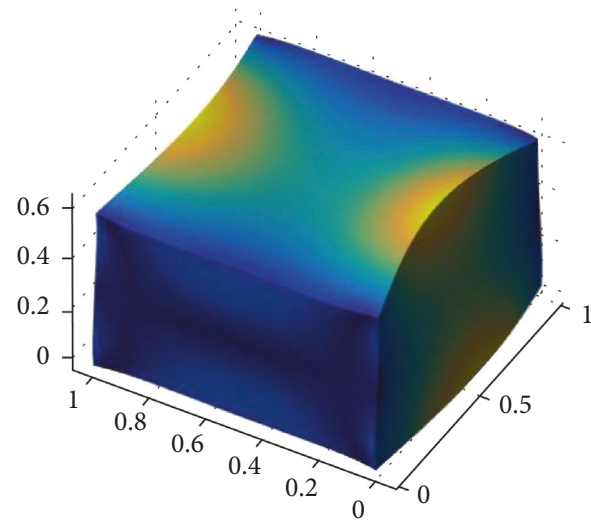

(e)

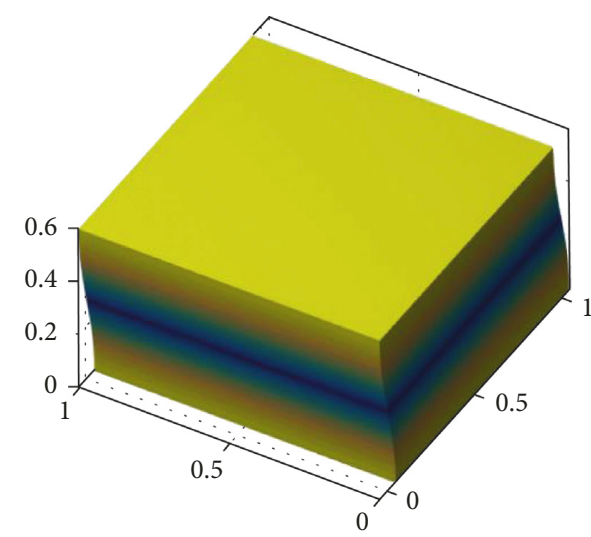

(f)

FIGURE 8: Mode shapes of orthotropic rectangular plates with the SFSF boundary condition: (a) 1st mode; (b) 2nd mode; (c) 3rd mode; (d) 4th mode; (e) 5th mode; (f) 6th mode.

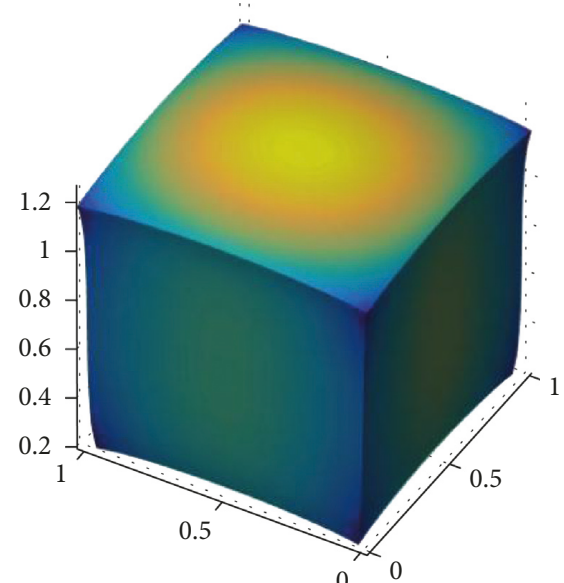

(a)

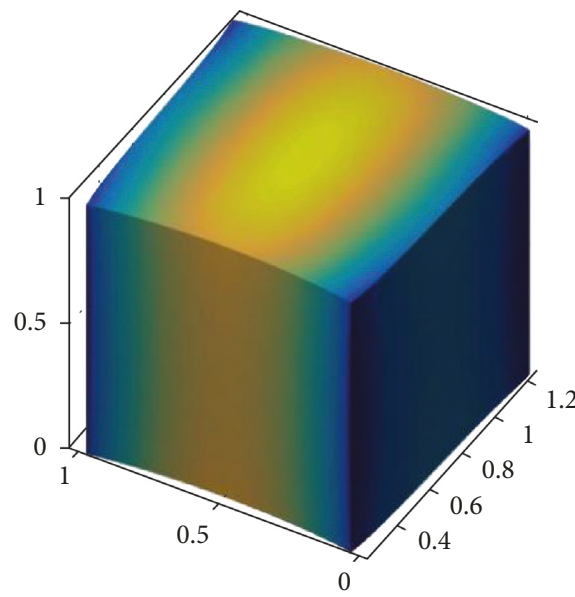

(c)

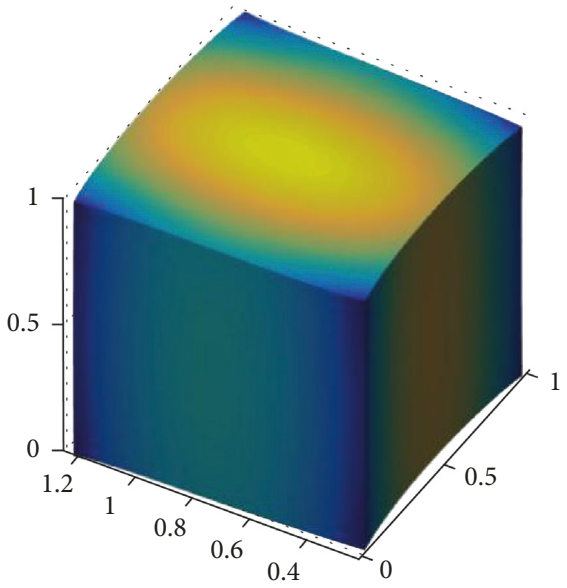

(b)

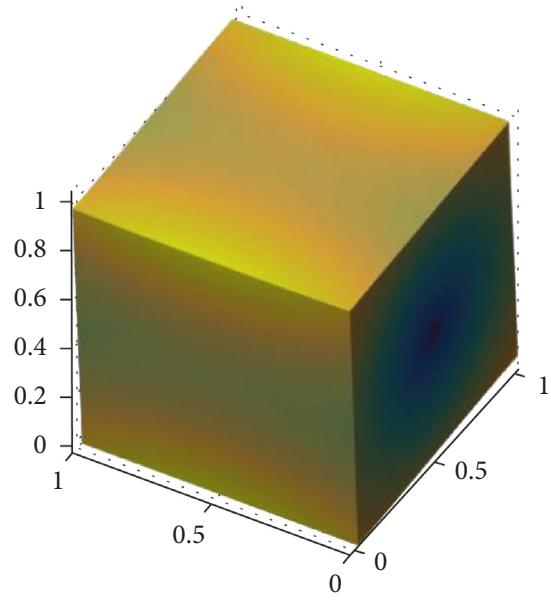

(d)

Figure 9: Continued. 


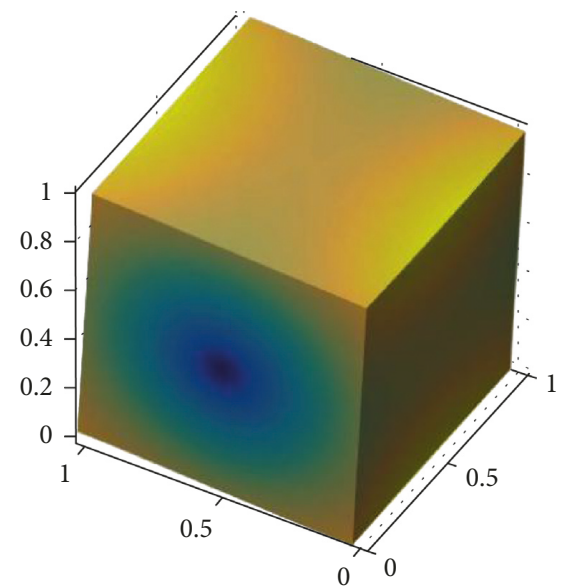

(e)

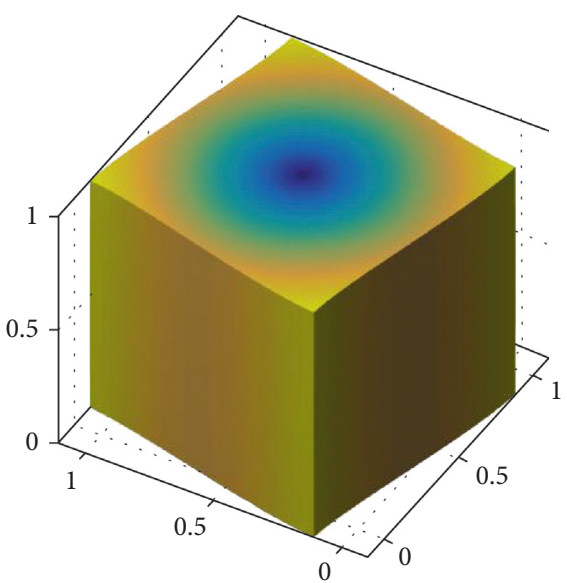

(f)

Figure 9: Mode shapes of orthotropic rectangular plates with the E4E4E4E4 boundary condition: (a) 1st mode; (b) 2nd mode; (c) 3rd mode; (d) 4th mode; (e) 5th mode; (f) 6th mode.

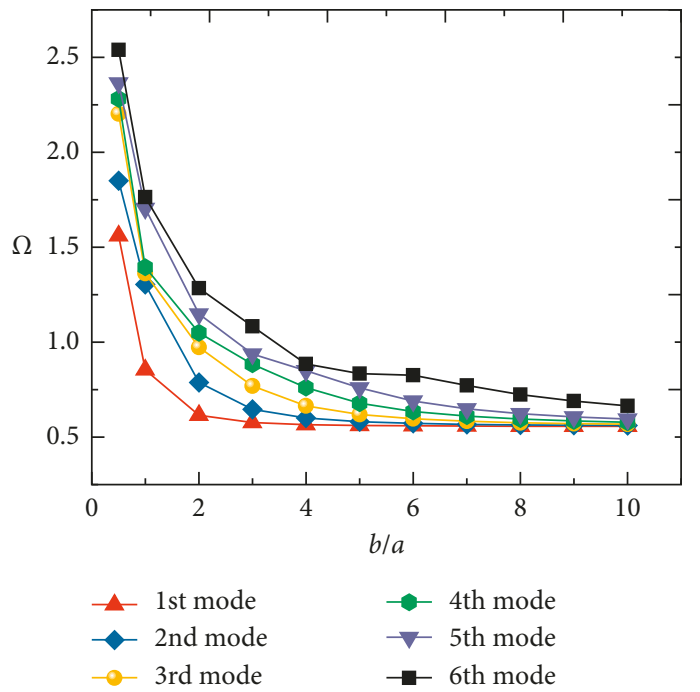

(a)

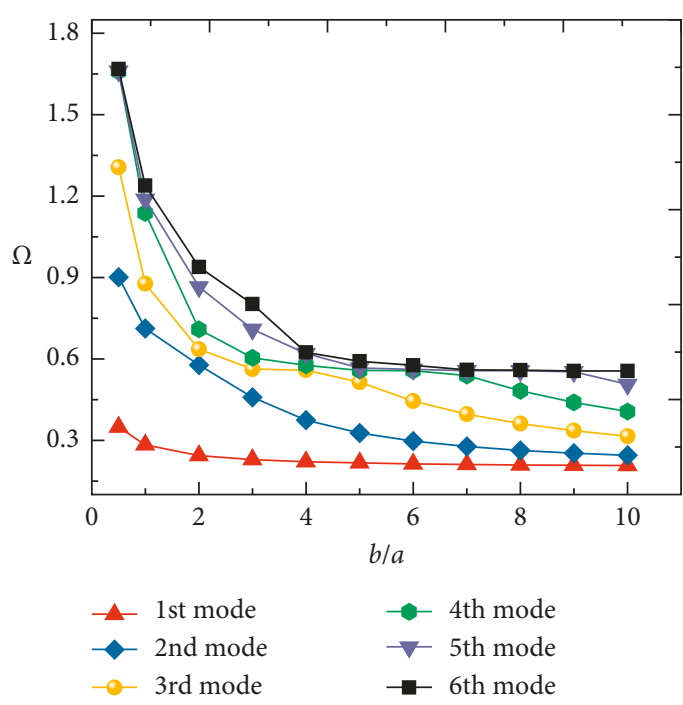

(c)

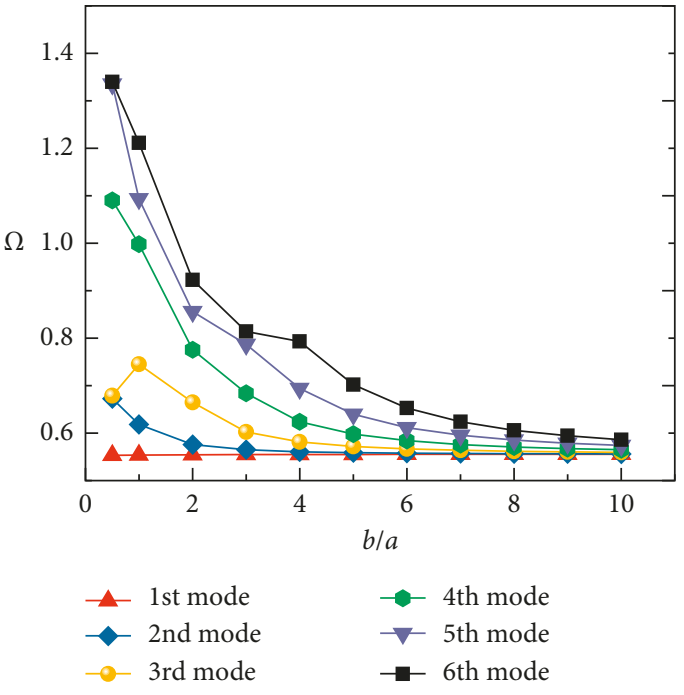

(b)

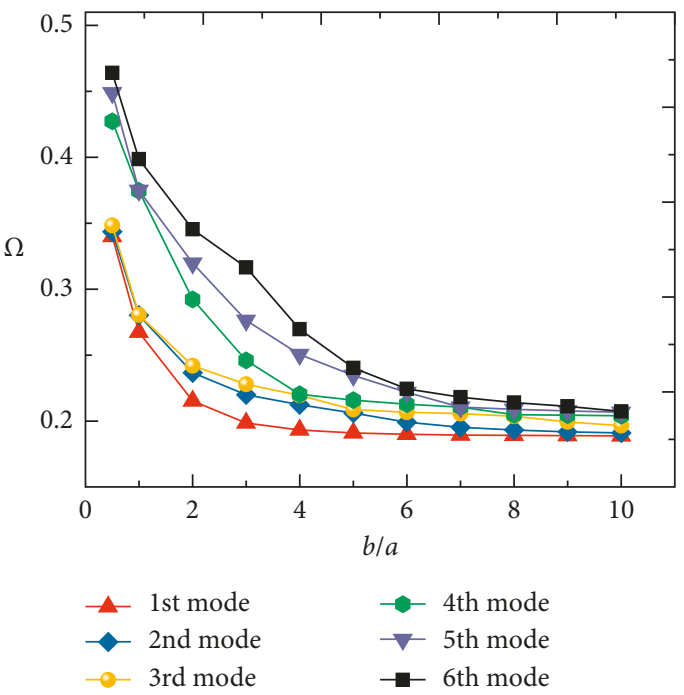

(d)

FIGURE 10: Variations of nondimensional frequency parameter $\Omega$ of orthotropic rectangular plates with respect to different aspect ratios $b / a$ : (a) CCCC; (b) CFCF; (c) E2E2E2E2; (d) E4E4E4E4. 

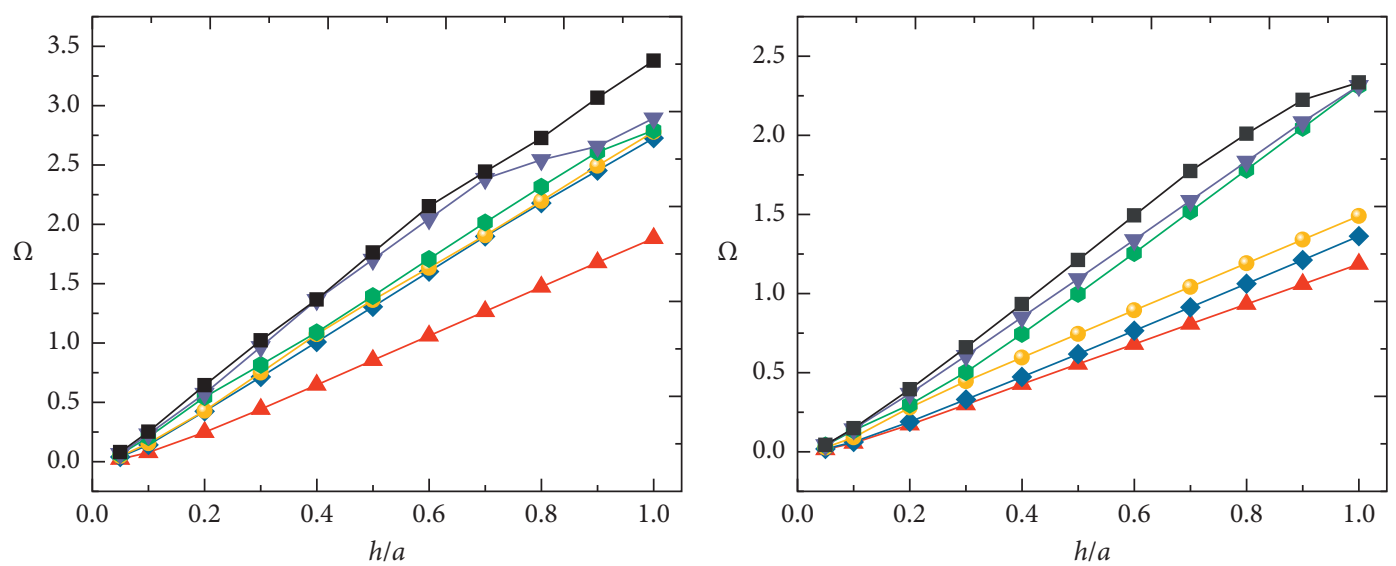

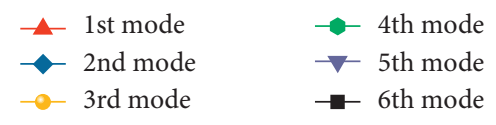

(a)

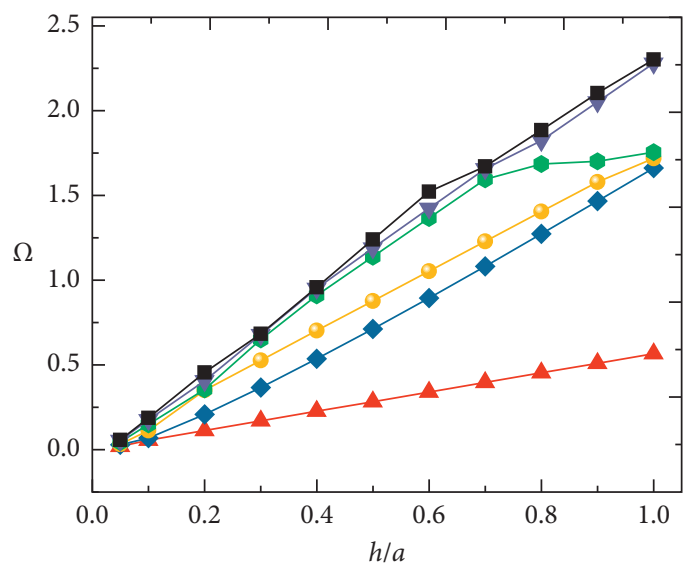

$\begin{array}{ll}- \text { 1st mode } & - \text { 4th mode } \\ - \text { 2nd mode } & -5 \text { th mode } \\ -- \text { 3rd mode } & -6 \text { th mode }\end{array}$

(c)

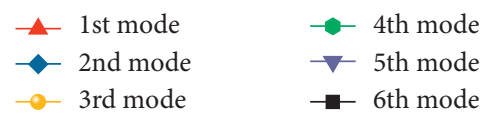

(b)

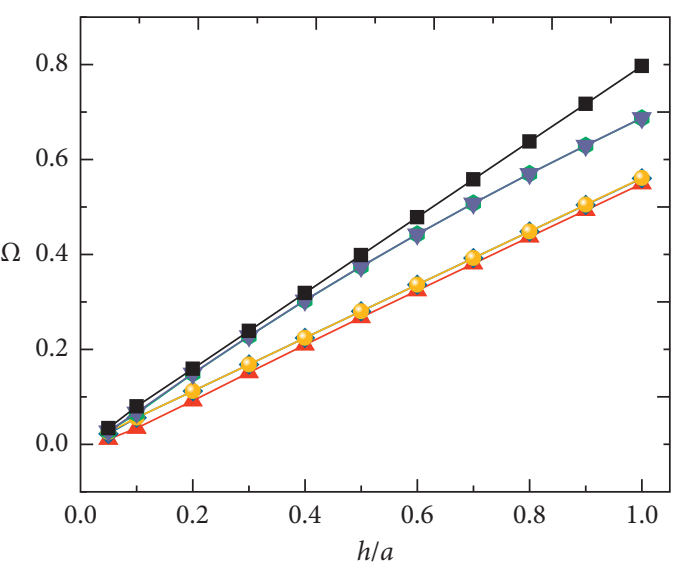

$\begin{array}{ll}- \text { 1st mode } & - \text { 4th mode } \\ - \text { 2nd mode } & -5 \text { th mode } \\ - \text { 3rd mode } & - \text { 6th mode }\end{array}$

(d)

FIGURE 11: Variations of nondimensional frequency parameter $\Omega$ of orthotropic rectangular plates with respect to different thickness ratios h/a: (a) CCCC; (b) CFCF; (c) E2E2E2E2; (d) E4E4E4E4.
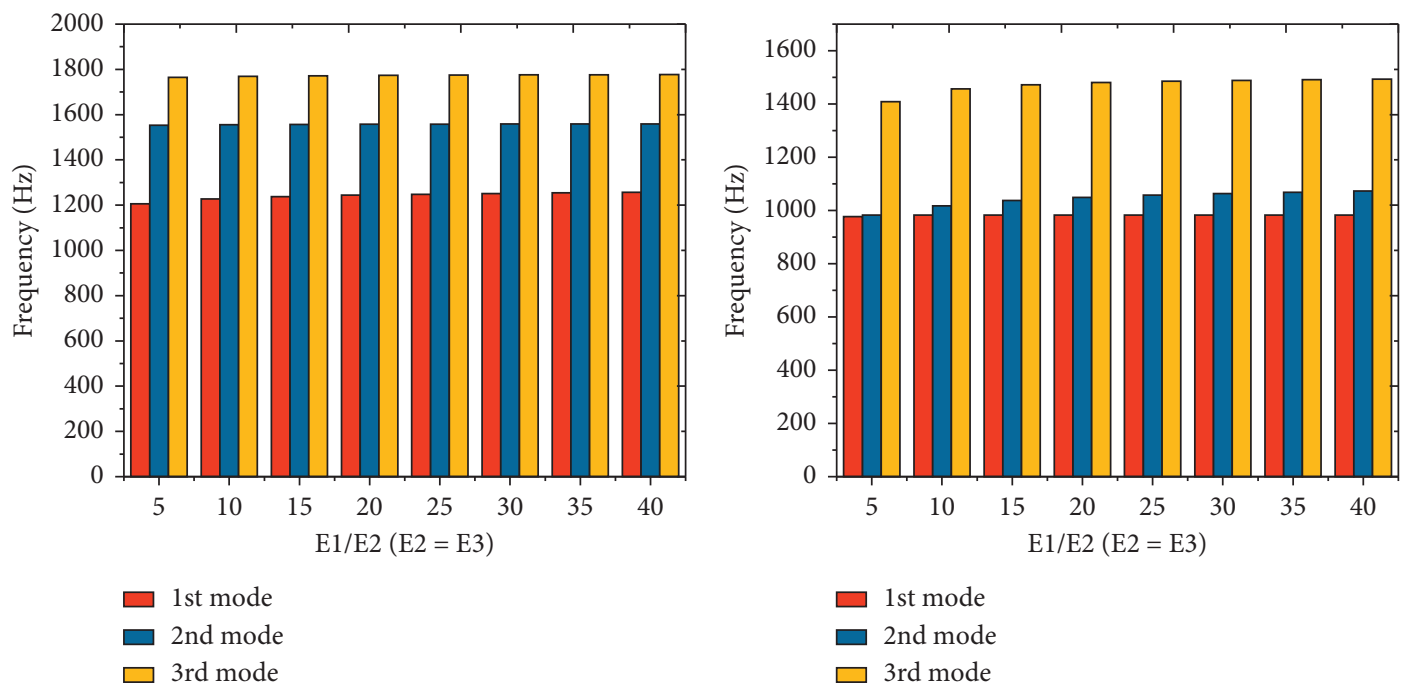

(a)

Figure 12: Continued. 


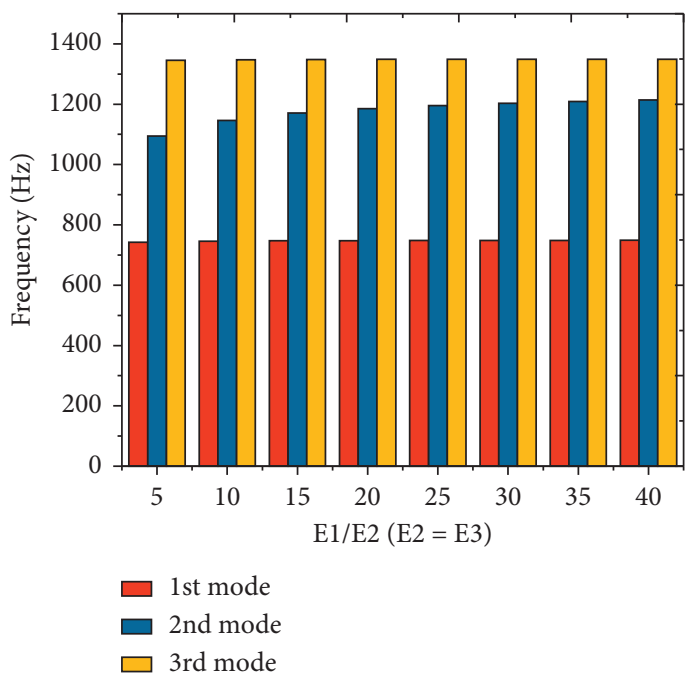

(c)

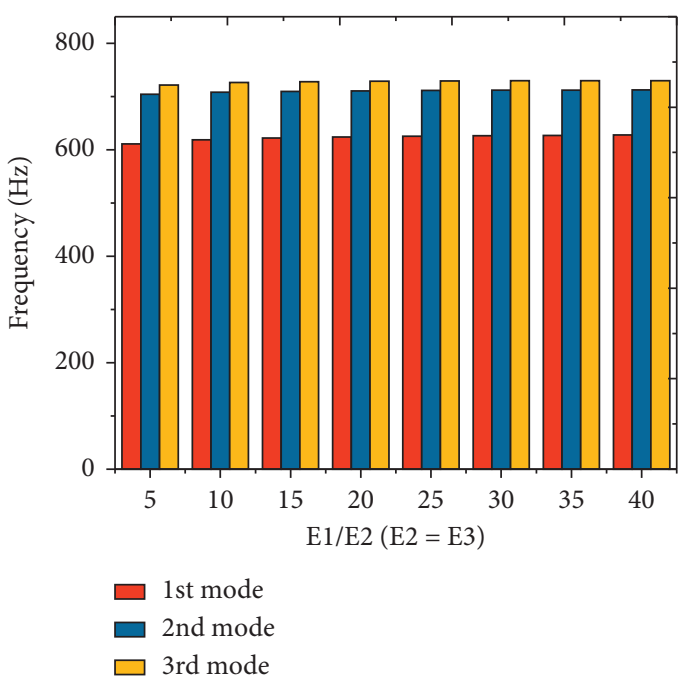

(d)

Figure 12: Variation of the first ten frequencies (Hz) against material parameter $\eta(\eta=\mathrm{E} 11 / \mathrm{E} 22)$ for a thick plate with different boundary conditions: (a) CCCC; (b) SSSS; (c) E1E1E1E1; (d) E4E4E4E4.

The matrix form can also be used to describe the equation:

$$
\left(\mathbf{K}-\omega^{2} \mathbf{M}\right) \mathbf{X}=\mathbf{0},
$$

where $\mathbf{K}$ and $\mathbf{M}$ indicate the stiffness matrices and mass matrices of the FGP annular and sector plate. And $\mathbf{X}$ is the sealed Fourier coefficient vector that is described as $\mathbf{P}=\left[\begin{array}{lll}\mathbf{A}_{m n l} & \mathbf{B}_{m n l} & \mathbf{C}_{m n l}\end{array}\right]^{\mathrm{T}}$. The two important parameters natural frequency and associated mode shape about thick orthotropic plates are acquired by the generalized eigenvalues of equation (16).

\section{Numerical Results and Discussion}

A series of numerical studies are carried out in this section, mainly including the following contents: (a) firstly, the convergence of this method is studied, the correctness is verified, and the definition of boundary conditions is studied; (b) secondly, accuracy of the method is validated by comparing with the relevant literature and datum; and (c) on this basis, the vibration performance of thick orthotropic plates which have arbitrary boundary conditions is further researched. In identifying the boundary conditions, these three symbols $\mathrm{F}, \mathrm{C}$, and $\mathrm{S}$ represent three boundary conditions, namely, free, clamping, and simple supported boundary conditions. And all the calculation codes are based on the MATLAB 8.2 platform. In addition, if no special declaration is made, the frequency dimensionless formula is defined as $\Omega=\omega \sqrt{\rho h^{2} / C_{11}}$.

3.1. Convergence and Stiffness Value Study. As we expected, as an excellent numerical method, it depends on its convergence and numerical constancy. However, it is found that the convergence and numerical constancy of proposed solution depend on the truncation number of admissible displacement functions (equations (10a)-(10k)). Therefore, it is important to research the convergence property and numerical constancy about these admissible displacements functions (equations (10a)-(10k)). The convergence of frequency parameters $\Omega$ about the orthotropic plate, which own different censoring numbers, is plotted in Table 1 . Three kinds of thickness, i.e., $h / a=0.1$ (thin plate), $h / a=0.3$ (moderately thick plate), and $h / a=0.5$ (thick plate), and seven types of truncated number, i.e., $M * N * Q=4 * 4 * 4$, $5 * 5 * 5, \quad 6 * 6 * 6, \quad 7 * 7 * 7, \quad 8 * 8 * 8, \quad 9 * 9 * 9, \quad$ and $10 * 10 * 10$, are examined in this table. The material property of this table is the aragonite crystal, and the specific material information is as follows: $C_{22} / C_{11}=0.543103, C_{12} /$ $C_{11}=0.23319, C_{33} / C_{11}=0.530172, C_{13} / C_{11}=0.010776, C_{23} /$ $C_{11}=0.098276, C_{66} / C_{11}=0.262931, C_{55} / C_{11}=0.159914$, and $C_{44} / C_{11}=0.26681$. The information in the table is available that no matter how thick the plate is, its natural frequency tends to stabilize rapidly when the number of truncation $M * N * Q$ increases. Especially when the truncated number value is more than $7 * 7 * 7$, the calculation result can keep the 4 digit effective number unchanged. Therefore, at this point, we can think that the calculation results have stabilized steadily. And the $M * N * Q$ representing truncated values of the revised sequence under the continuation of numerical examples is defined as $M * N * Q=8 * 8 * 8$.

In this paper, we aim to establish an analytical model for vibration of orthotropic plates under the conditions of the arbitrary boundary by the method of arranging three kinds of linear springs at the edges. In addition, the simulation of the clamped boundary conditions is to set the spring stiffness value to infinity, and in practical cases, numerical instability will occur when the stiffness value is set to infinity due to the limitation of computational resources. Therefore, we must use a finite value to replace the infinite stiffness value. Thus, the vibration performances on orthotropic plates are affected with the elastic boundary. 
Frequency parameters $\Omega$ of the elastically constrained orthotropic plate are summarized in Tables 2-4. Three elastic boundary conditions are defined in the following ways: (1) elastically restrained in the $x$ direction (Table 2), $k_{x 0}^{v}=k_{x 0}^{w}=0, k_{x 0}^{u}=10^{2} \sim 10^{18}$; (2) elastically restrained in the $y$ direction (Table 3), $k_{x 0}^{u}=k_{x 0}^{w}=0, k_{x 0}^{v}=10^{2} \sim 10^{18}$; and (3) elastically restrained in the $z$ direction (Table 4), $k_{x 0}^{u}=k_{x 0}^{v}=0, k_{x 0}^{w}=10^{2} \sim 10^{18}$. To simplify the research, the $y=$ constant boundary is a free boundary, one end of orthotropic plates $(x=a)$ is a clamped boundary condition, and the other edge $x=0$ is elastically constrained. Through the comparison of three tabular data, the results show that the $k_{x 0}^{w}$ parameters have the greatest effect with the vibration behavior of orthotropic plates, followed by $k_{x 0}^{u}$ and finally the $k_{x 0}^{v}$ parameter. This phenomenon can also be found directly from the modal shapes in Figures 2-4. Based on the above research, we further define three conditions with the classical boundary and four conditions of the elastic boundary in the following example, and the detailed spring stiffness coefficient is shown in Table 5.

\subsection{Vibration Analysis of Thick Orthotropic Plates with} Various Boundary Conditions. As mentioned before, there are relatively few studies on this topic. Therefore, in order to dig deeper into the details of the research content, the author will further carry out the study of vibration analysis with these thick orthotropic plates of various boundary conditions. The numerical results of a thick plate with different boundary conditions compared with published literature [26] are shown in Table 6. It can be easy to find that the maximum error between the present method and literature data is no more than $0.15 \%$. So, it can be concluded that the present method is accurate to solve the vibration behaviors of thick plates with various boundary conditions.

The free vibration experiments of the isotropic plate structure was carried out to verify the feasibility of the present method, and the material properties are chosen as $E=210 \mathrm{GPa}, \rho=7850 \mathrm{~kg} / \mathrm{m} 3, \quad \mu=0.3, \quad a=b=1.2 \mathrm{~m}$, and $h=0.008 \mathrm{~m}$. The test was carried out by loading on multiple excitation points; meanwhile, the responding signals were collected from a single point. The comparison of natural frequencies which are obtained by the present method, experiment, and FEM for the isotropic plate is shown in Table 7. It is easy to find that the results achieved by three different approaches are almost infallible, and the maximum error is less than 2\%. Figure 5 exhibits the test system and model. In order to better understand the vibration characteristics of the isotropic plate, the first three mode shapes obtained by experiment are shown in Figure 6. It can be concluded that the presented method can solve the vibration behaviors of isotropic plates.

Tables $8-10$ provide frequency parameters $\Omega$ belonging to the first eight bits of the orthotropic plate under different boundary conditions, and these different boundary conditions mentioned above include four kinds of classical boundary conditions and four groups of elastic boundary conditions. In this above table, the material parameters and aspect ratios $a / b=1$ of the orthotropic plate are consistent with those in Table 1 and the thickness ratios are $h / a=0.3$ (Table 8), $h / a=0.6$ (Table 9), and $h / a=1$ (Table 10 ), respectively. As can be seen from the table above, frequency parameters $\Omega$ obtained under the boundary conditions of CCCC are the largest, while the frequency parameter obtained under the E4E4E4E4 boundary condition is the smallest. Therefore, it is reflected that the increase of boundary stiffness of the orthotropic plate will result in the increase of frequency parameters. For the sake of better show the advantages of this method, Figures 7-9 provide threedimensional modal shapes with the orthotropic plate under different boundary conditions. Unlike other methods, only two-dimensional modal shapes can be drawn, so the vibration characteristics of the orthotropic plate can be more clearly displayed. Lastly, the influence of aspect ratio $b / a$ and thickness ratio $h / a$ is also reported. The change regulation about nondimensional frequency parameter $\Omega$ of the orthotropic rectangular plates under the different aspect ratio $b / a$ is shown in Figure 10. There are four sets of boundary conditions CCCC, CFCF, $\mathrm{E} 2 \mathrm{E} 2 \mathrm{E} 2 \mathrm{E} 2$, and E4E4E4E4 which are used in this example. From these pictures, it can be clearly detected that the frequency parameters of orthotropic rectangular plates gradually decrease as the increase of aspect ratio al $b$, and it need to be noticed that the frequency parameters almost remain unchanged when the aspect ratio increases to a critical aspect ratio. Moreover, it is found that the critical aspect ratio is directly related to the boundary conditions. Figure 11 shows the relation between the nondimensional frequency parameter $\Omega$ and the thickness ratio $h / a$ of orthotropic rectangular plates. From this diagram, it becomes apparent that the frequency parameters on orthotropic rectangular plates rise monotonously with the raise of thickness ratio $h / a$, regardless of the boundary conditions or modal order. Finally, the influence of material properties on structural vibration characteristics is carried out. Figure 12 shows the histogram of the variation of the first three modes frequency parameters of the structure with different orthogonal stiffness ratios E11/E22 under different boundary conditions. The material parameters are defined as follows: $E_{y}=E_{z}=8.963 \mathrm{GPa}, E_{x} / E_{y}=5,10,15,20,25,30,35$, and $40 ; G_{x y}=6.205 \mathrm{GPa}, G_{x z}=7.101 \mathrm{GPa}$, and $G_{y z}=6.205 \mathrm{GPa}$; $\mu_{x y}=0.3, \mu_{y z}=0.49$, and $\mu_{x z}=0.3$; and $\rho=1605 \mathrm{~kg} / \mathrm{m}^{3}$. The geometric parameters are defined as follows: $a=b=1 \mathrm{~m}$ and $h=0.5 \mathrm{~m}$. It can be clearly found from Figure 12 that the frequency parameters of the structure always increase with the increase of the orthotropic stiffness value no matter what the boundary conditions of the structure are, and the trend is more obvious at the stage of smaller stiffness. Through the above discussion and study, it is easy to discover that the vibration behavior of the orthotropic rectangular plates is related to both geometric parameters and boundary conditions.

\section{Conclusions}

This paper analyzed the free vibration of orthotropic plates under arbitrary boundary conditions by semianalytical 
three-dimensional elastic solution. And 3D elastic theory is adopted to achieve the energy functional of orthotropic plates. The real boundary conditions of orthotropic plates are simulated by uniformly arranging three groups of linear springs on the edges of plates. The admissible displacement functions of orthotropic plates are consistently expanded as a modified Fourier series that it is based on 3D Fourier cosine series and auxiliary function. Under this framework, when the boundary conditions of the orthotropic plates change, just only the boundary parameters need to be modified and do not need a new derivation, thus greatly saving the modeling time. The astringency, accuracy, and constancy of the method of this paper are validated by means of series of numerical examples. Moreover, some new results and parameters of orthotropic plates under different boundary conditions, thickness ratios, and aspect ratios are also presented which can be used as a benchmark to help subsequent scholars engage in such research. At the same time, the results of this paper can be used as reference data for future researches in this field.

\section{Data Availability}

The data used to support the findings of this study are available from the corresponding author upon request.

\section{Conflicts of Interest}

The authors declare that they have no conflicts of interest.

\section{Acknowledgments}

This study was supported financially by the National Natural Science Foundation of China (Grant nos. 51109101, 51779111, and 51622902), the Key University Science Research Project of Jiangsu Province (Grant no. 17KJA416003), and the Key Laboratory Foundation of Jiangsu Province (Grant no. CJ1601).

\section{References}

[1] C.-P. Wu and Y.-C. Liu, "A review of semi-analytical numerical methods for laminated composite and multilayered functionally graded elastic/piezoelectric plates and shells," Composite Structures, vol. 147, pp. 1-15, 2016.

[2] A. S. Sayyad and Y. M. Ghugal, "On the free vibration analysis of laminated composite and sandwich plates: a review of recent literature with some numerical results," Composite Structures, vol. 129, pp. 177-201, 2015.

[3] A. S. Sayyad and Y. M. Ghugal, "Bending, buckling and free vibration of laminated composite and sandwich beams: a critical review of literature," Composite Structures, vol. 171, pp. 486-504, 2017.

[4] H.-T. Thai and S.-E. Kim, "Levy-type solution for free vibration analysis of orthotropic plates based on two variable refined plate theory," Applied Mathematical Modelling, vol. 36, no. 8, pp. 3870-3882, 2012.

[5] H.-T. Thai and S.-E. Kim, "Levy-type solution for buckling analysis of orthotropic plates based on two variable refined plate theory," Composite Structures, vol. 93, no. 7, pp. 17381746, 2011.
[6] H.-T. Thai and S.-E. Kim, "Analytical solution of a two variable refined plate theory for bending analysis of orthotropic levy-type plates," International Journal of Mechanical Sciences, vol. 54, no. 1, pp. 269-276, 2012.

[7] K. Sepahvand, S. Marburg, and H.-J. Hardtke, "Stochastic free vibration of orthotropic plates using generalized polynomial chaos expansion," Journal of Sound and Vibration, vol. 331, no. 1, pp. 167-179, 2012.

[8] B. Abbès and Y. Q. Guo, "Analytic homogenization for torsion of orthotropic sandwich plates: application to corrugated cardboard," Composite Structures, vol. 92, no. 3, pp. 699-706, 2010.

[9] Y. M. Ghugal and A. S. Sayyad, "Free vibration of thick orthotropic plates using trigonometric shear deformation theory," Latin American Journal of Solids and Structures, vol. 8, no. 3, pp. 229-243, 2011.

[10] M.-H. Hsu, "Vibration analysis of orthotropic rectangular plates on elastic foundations," Composite Structures, vol. 92, no. 4, pp. 844-852, 2010.

[11] E. Bahmyari and A. Rahbar-Ranji, "Free vibration analysis of orthotropic plates with variable thickness resting on nonuniform elastic foundation by element free Galerkin method," Journal of Mechanical Science and Technology, vol. 26, no. 9, pp. 2685-2694, 2012.

[12] N. Valizadeh, T. Q. Bui, V. T. Vu, H. T. Thai, and M. N. Nguyen, "Isogeometric simulation for buckling, free and forced vibration of orthotropic plates," International Journal of Applied Mechanics, vol. 5, no. 2, article 1350017, 2013.

[13] Y. F. Xing and T. F. Xu, "Solution methods of exact solutions for free vibration of rectangular orthotropic thin plates with classical boundary conditions," Composite Structures, vol. 104, pp. 187-195, 2013.

[14] A. M. Zenkour, "Bending of orthotropic plates resting on Pasternak's foundations using mixed shear deformation theory," Acta Mechanica Sinica, vol. 27, no. 6, pp. 956-962, 2011.

[15] B. Bakhadda, M. B. Bouiadjra, F. Bourada et al., "Dynamic and bending analysis of carbon nanotube-reinforced composite plates with elastic foundation," Wind and Structures, vol. 27, no. 5, pp. 311-324, 2018.

[16] F. Bourada, A. A. Bousahla, M. Bourada et al., "Dynamic investigation of porous functionally graded beam using a sinusoidal shear deformation theory," Wind and Structures, vol. 28, no. 1, pp. 19-30, 2019.

[17] A. Boukhari, H. A. Atmane, A. Tounsi, E. A. Adda Bedia, and S. R. Mahmoud, "An efficient shear deformation theory for wave propagation of functionally graded material plates," Structural Engineering and Mechanics, vol. 57, no. 5, pp. 837-859, 2016.

[18] H. Fourn, H. A. Atmane, M. Bourada et al., "A novel four variable refined plate theory for wave propagation in functionally graded material plates," Steel and Composite Structures, vol. 27, no. 1, pp. 109-122, 2018.

[19] S. O. Papkov and J. R. Banerjee, "A new method for free vibration and buckling analysis of rectangular orthotropic plates," Journal of Sound and Vibration, vol. 339, pp. 342-358, 2015.

[20] M.-F. Liu, T.-P. Chang, and Y.-H. Wang, "Free vibration analysis of orthotropic rectangular plates with tapered varying thickness and winkler spring foundation," Mechanics Based Design of Structures and Machines, vol. 39, no. 3, pp. 320-333, 2011. 
[21] D. Shi, Q. Wang, X. Shi, and F. Pang, "A series solution for the in-plane vibration analysis of orthotropic rectangular plates with non-uniform elastic boundary constraints and internal line supports," Archive of Applied Mechanics, vol. 85, no. 1, pp. 51-73, 2015.

[22] A. A. Jafari and S. A. Eftekhari, "An efficient mixed methodology for free vibration and buckling analysis of orthotropic rectangular plates," Applied Mathematics and Computation, vol. 218, no. 6, pp. 2670-2692, 2011.

[23] B. Liu and Y. Xing, "Exact solutions for free vibrations of orthotropic rectangular Mindlin plates," Composite Structures, vol. 93, no. 7, pp. 1664-1672, 2011.

[24] B. Liu and Y. Xing, "Comprehensive exact solutions for free in-plane vibrations of orthotropic rectangular plates," European Journal of Mechanics-A/Solids, vol. 30, no. 3, pp. 383-395, 2011.

[25] H.-H. Chang and J.-Q. Tarn, "Three-dimensional elasticity solutions for rectangular orthotropic plates," Journal of Elasticity, vol. 108, no. 1, pp. 49-66, 2012.

[26] T. M. Teo and K. M. Liew, "Three-dimensional elasticity solutions to some orthotropic plate problems," International Journal of Solids and Structures, vol. 36, no. 34, pp. 5301-5326, 1999.

[27] A. Tian, R. Ye, and Y. Chen, "A new higher order analysis model for sandwich plates with flexible core," Journal of Composite Materials, vol. 50, no. 7, pp. 949-961, 2016.

[28] A. Tian, R. Ye, P. Ren et al., "New higher-order models for sandwich plates with a flexible core and their accuracy assessment," International Journal of Structural Stability and Dynamics, vol. 19, no. 3, article 1950024, 2019.

[29] H. Li, F. Pang, X. Miao, S. Gao, and F. Liu, "A semi analytical method for free vibration analysis of composite laminated cylindrical and spherical shells with complex boundary conditions," Thin-Walled Structures, vol. 136, pp. 200-220, 2019.

[30] H. Li, F. Pang, and H. Chen, "A semi-analytical approach to analyze vibration characteristics of uniform and stepped annular-spherical shells with general boundary conditions," European Journal of Mechanics-A/Solids, vol. 74, pp. 48-65, 2019.

[31] H. Li, F. Pang, H. Chen, and Y. Du, "Vibration analysis of functionally graded porous cylindrical shell with arbitrary boundary restraints by using a semi analytical method," Composites Part B: Engineering, vol. 164, pp. 249-264, 2019.

[32] H. H. Abdelaziz, M. A. A. Meziane, A. A. Bousahla et al., "An efficient hyperbolic shear deformation theory for bending, buckling and free vibration of FGM sandwich plates with various boundary conditions," Steel and Composite Structures, vol. 25, no. 6, pp. 693-704, 2017.

[33] B. Karami, M. Janghorban, and A. Tounsi, "Galerkin's approach for buckling analysis of functionally graded anisotropic nanoplates/different boundary conditions," Engineering with Computers, 2018.

[34] M. A. A. Meziane, H. H. Abdelaziz, and A. Tounsi, "An efficient and simple refined theory for buckling and free vibration of exponentially graded sandwich plates under various boundary conditions," Journal of Sandwich Structures \& Materials, vol. 16, no. 3, pp. 293-318, 2014.

[35] J. Cui, R. Ye, N. Zhao, J. Wu, and M. Wang, “Assessment on energy absorption of double layered and sandwich plates under ballistic impact," Thin-Walled Structures, vol. 130, pp. 520-534, 2018.

[36] K. Liu, B. Liu, R. Villavicencio, Z. Wang, and C. Guedes Soares, "Assessment of material strain rate effects on square steel plates under lateral dynamic impact loads," Ships and Offshore Structures, vol. 13, no. 2, pp. 217-225, 2018.

[37] H. Li, F. Pang, X. Miao, and Y. Li, "Jacobi-Ritz method for free vibration analysis of uniform and stepped circular cylindrical shells with arbitrary boundary conditions: a unified formulation," Computers \& Mathematics with Applications, vol. 77, no. 2, pp. 427-440, 2019.

[38] H. Zhang, D. Shi, S. Zha, and Q. Wang, "Vibro-acoustic analysis of the thin laminated rectangular plate-cavity coupling system," Composite Structures, vol. 189, pp. 570-585, 2018.

[39] H. Zhang, D. Shi, S. Zha, and Q. Wang, "Parameterization study on the moderately thick laminated rectangular platecavity coupling system with uniform or non-uniform boundary conditions," Composite Structures, vol. 194, pp. 537-554, 2018.

[40] H. Zhang, D. Shi, S. Zha, and Q. Wang, "A simple first-order shear deformation theory for vibro-acoustic analysis of the laminated rectangular fluid-structure coupling system," Composite Structures, vol. 201, pp. 647-663, 2018.

[41] J. Zhao, F. Xie, A. Wang, C. Shuai, J. Tang, and Q. Wang, "Vibration behavior of the functionally graded porous (FGP) doubly-curved panels and shells of revolution by using a semianalytical method," Composites Part B: Engineering, vol. 157, pp. 219-238, 2019. 


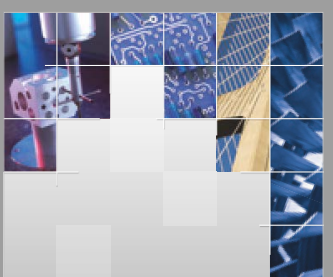

\section{Enfincering}
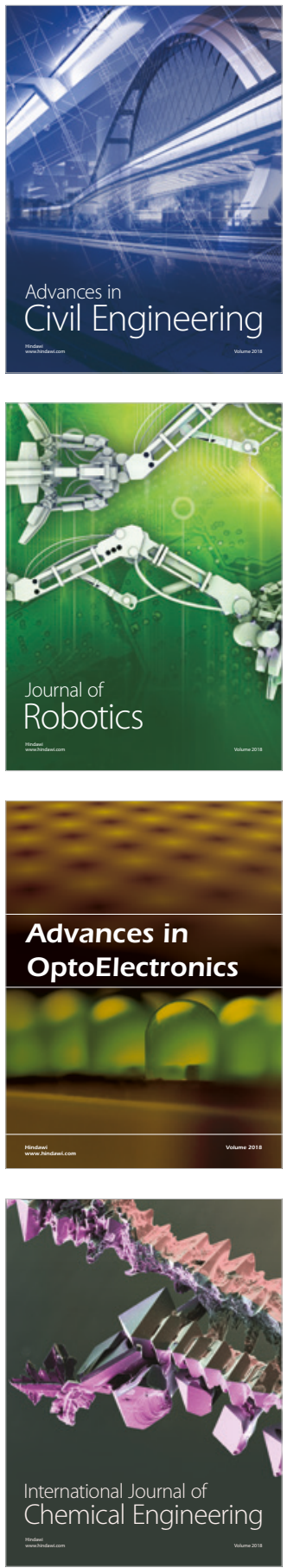

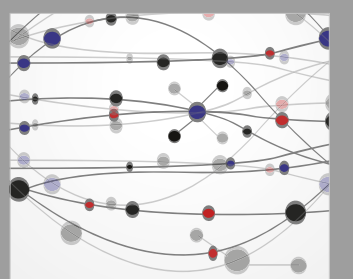

\section{Rotating \\ Machinery}

The Scientific World Journal

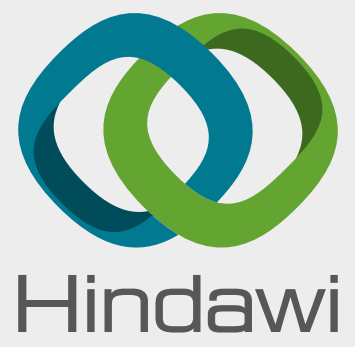

Submit your manuscripts at

www.hindawi.com
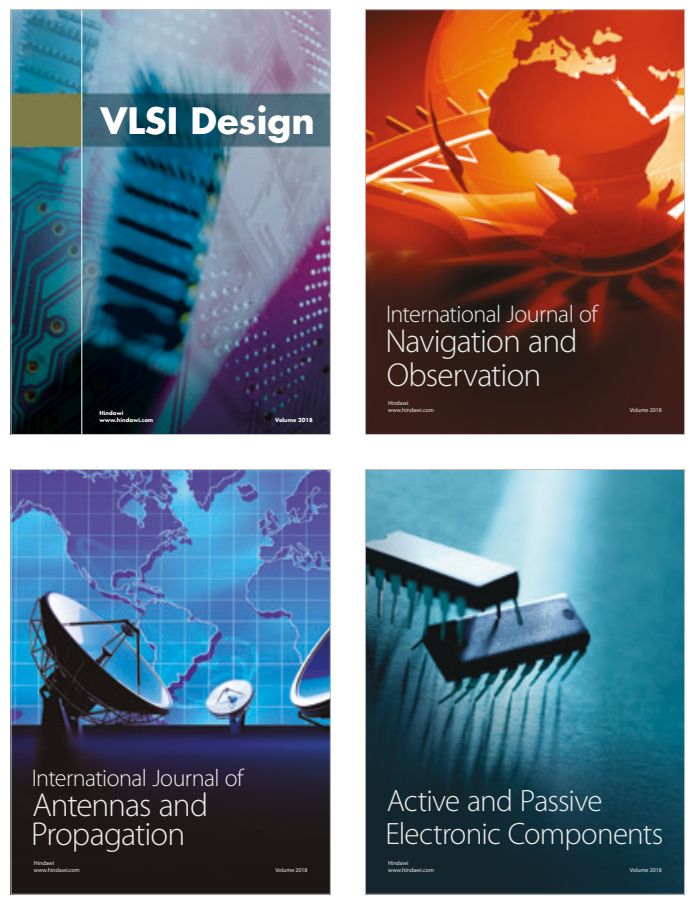
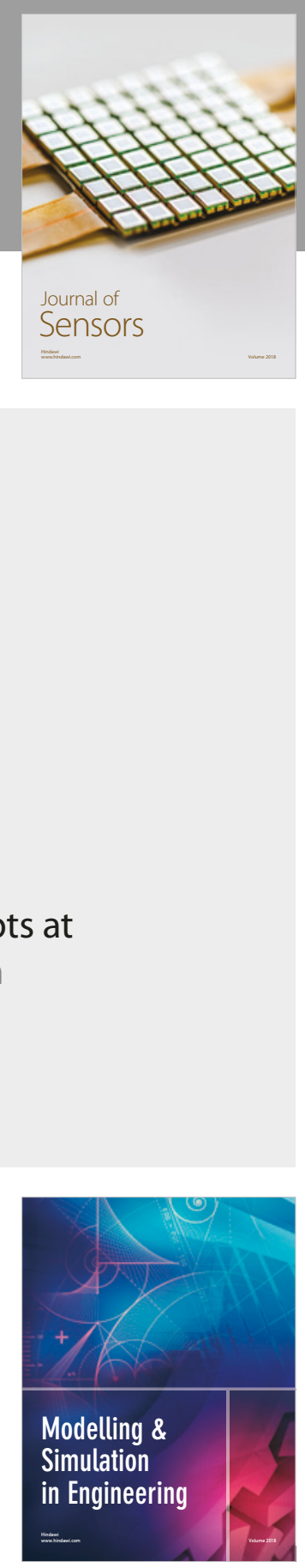

\section{Advances \\ Multimedia}
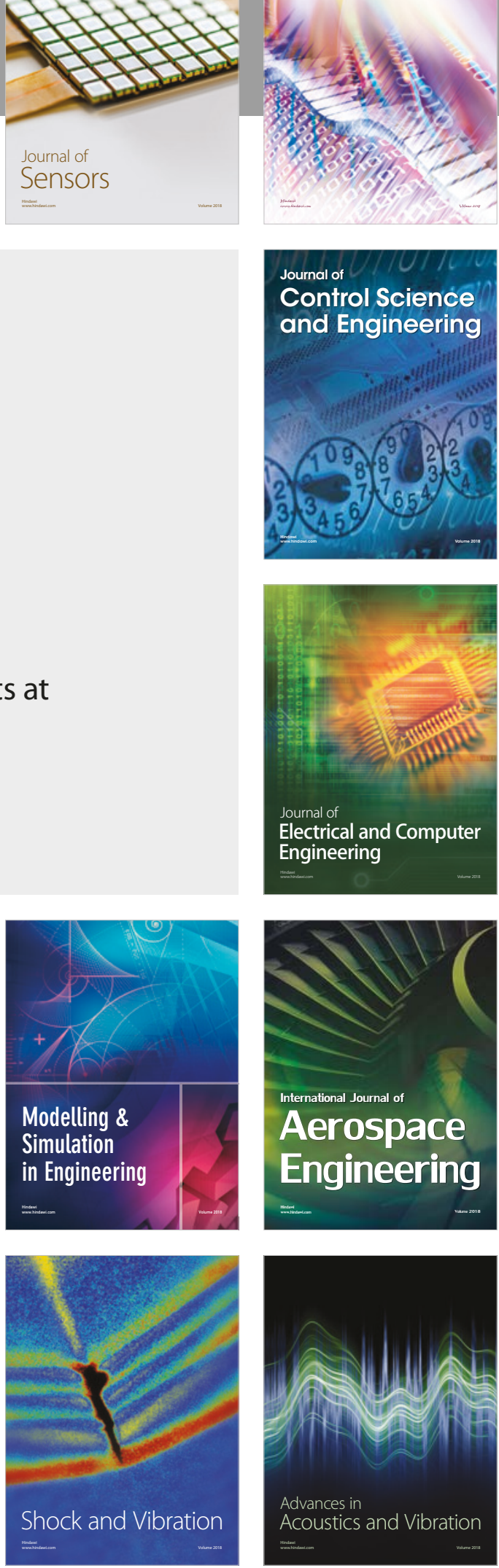